\title{
Mesoporous Silica Nanoparticles: Their Projection in Nanomedicine
}

\author{
María Vallet-Regí \\ Departamento de Química Inorgánica y Bioinorgánica, Facultad de Farmacia, UCM, Centro de Investigación Biomédica en Red, \\ Bioingeniería, Biomateriales y Nanomedicina (CIBER-BBN), 28040 Madrid, Spain \\ Correspondence should be addressed to María Vallet-Regí, vallet@farm.ucm.es
}

Received 26 June 2012; Accepted 24 July 2012

Academic Editors: M. Leoni and Y. Masuda

Copyright ( 2012 María Vallet-Regí. This is an open access article distributed under the Creative Commons Attribution License, which permits unrestricted use, distribution, and reproduction in any medium, provided the original work is properly cited.

\begin{abstract}
Mesoporous silica nanoparticles are receiving growing attention by the scientific biomedical community. Among the different types of inorganic nanomaterials, mesoporous silica nanoparticles have emerged as promising multifunctional platforms for nanomedicine. Since their introduction in the drug delivery landscape in 2001, mesoporous materials for drug delivery are receiving growing scientific interest for their potential applications in the biotechnology and nanomedicine fields. The ceramic matrix efficiently protects entrapped guest molecules against enzymatic degradation or denaturation induced by $\mathrm{pH}$ and temperature as no swelling or porosity changes take place as a response to variations in the surrounding medium. It is possible to load huge amounts of cargo into the mesopore voids and capping the pore entrances with different nanogates. The application of a stimulus provokes the nanocap removal and triggers the departure of the cargo. This strategy permits the design of stimuliresponsive drug delivery nanodevices.
\end{abstract}

\section{Introduction}

Research in mesoporous materials is at the limelight since it has developed an exciting work in chemical synthesis responsible for an enormous amount of exciting practical applications for the welfare of society. From catalysis to medical and nanotechnological applications, there are uncountable new materials from the discovery of mesoporous solids. And, in this discovery, there are three main actors to be highlighted.

First of all, the makers and pioneers on mesoporous materials synthesis, which took place at the early nineties, and are the Japanese group headed by Kuroda [1] together with the group from Mobil Oil Corporation headed by Kresge $[2,3]$. Some years later, the research group of Terasaki [4-8] performed an excellent characterization that allowed to understand the behavior of these materials and to project them to new and important applications, as it is discussed elsewhere [9-12].

\section{Their Discovery}

During the 1970's, the chemistry in confined spaces carried out by the zeolites industry represented the origin of mesoporous materials thanks to the large amount of experimental work performed. Mobil Corporation developed in 1972 a synthetic path to convert methanol into gasoline using as catalyst the Zeolite ZSM-5 (Zeolite Socony Mobil). This work supposed the inspiration to find zeolites with bigger pore size in order to implement the previously mentioned conversion. The purpose of that research was to obtain cheaper gasoline from acid-base reactions taking place in the small cavities of those zeolites. This approach did not work using zeolites, and this drawback motivated the researchers to design materials with larger pores.

It was in this scenario where the porous solids industry was trying to find materials with larger pores than zeolites, which are microporous-type materials, when the first 
silica-based mesoporous material was produced almost simultaneously in the early 1990's by Mobil Corporation at USA [2] and Kuroda research team at Japan [1]. The combination of silica with amphiphilic surfactant (surface active agents) molecules leaded to the production of mesoporous silica materials with large pores. In this sense, researchers employed the surfactants as structure directing agents to produce M41S and KSW-n mesoporous materials, respectively.

However, the pioneer work by the American and Japanese groups in the early nineties was only the starting point of research in mesoporous solids, which has terrifically increased in the last 20 years. In fact, many research teams are focused in the production and evaluation at a fundamental level of the mechanisms involved in the formation of mesoporous materials. As a consequence, new reaction pathways have been found which lead to more complex nanostructures with a wide compositional range. Additionally, synthesis and characterization go in parallel with possible applications, which in the early days of mesoporous materials were based on catalytic applications and played a decisive role in the fast growing of mesoporous research area.

Chronologically, the most important landmarks in the development of ordered mesoporous materials can be summarized as follows.

(i) The starting work by the pioneers on silica mesophases.

(ii) The subsequent expansion and variation on the inorganic framework variation.

(iii) The introduction of novel polymeric surfactants with a higher degree of variability.

(iv) The introduction of nanocasting, which introduces a new dimension on ordered mesoporous materials technology.

Thus, it is crystalline clear that a whole new research area was opened by the work in the early nineties above mentioned, and it is going to be exciting to find out how many developments with their respective commercial applications will be achieved in the near future.

\section{Their Chemistry}

Ordered mesoporous materials are unique materials that are defined by an ordered and repetitive mesostructure of pores and disordered arrangement at the atomic level. Their synthesis is based on the use of surfactants, which are amphiphilic organic molecules that act as templates to direct the arrangement and subsequent condensation of the inorganic precursors. The whole process leads to a network of cavities arranged periodically. An important milestone in the development of mesoporous materials synthesis has been the advances in the sol-gel technology, which is based on inorganic polymerization reactions that take place under mild conditions, which permits the combination of inorganic phases with organic and/or biological systems. Thus, this sol-gel chemistry has allowed the introduction of mesoporosity to traditional ceramic compositions, leading to novel and interesting properties [13].
As a consequence of the templating method employed for the synthesis, the porosity properties of ordered mesoporous materials would depend on the type of surfactant employed during the liquid-crystal templating mechanism $[1,2]$.

In detail, the synthetic process is based on the dissolution of surfactant molecules into polar solvents to yield the socalled liquid crystals. The amount of surfactant dissolved in the solution plays a very important role during the synthetic process, and when the concentration is above the critical micelar concentration $(\mathrm{cmc})$, the surfactant molecules aggregate themselves to form micelles. Obviously, the characteristics of these micelles depend on the nature of the employed surfactant and/or the experimental conditions, for example, temperature, $\mathrm{pH}$, or concentration.

After micelles formation, those micelles aggregate themselves to yield supramicellar structures with determined geometries, which again will depend on the chemical nature of the surfactant and the processing conditions. The resulting mesoporous framework depends on these supramicellar geometries, such as hexagonal, cubic, and laminar.

Once the supramicellar aggregates are formed, the silica precursors, normally alkoxysilanes, are added to the so-called liquid crystals and the sol-gel chemistry starts to hydrolase and condensate those alkoxysilanes to yield highly condensed silicon oxide around that supramicellar mesostructure. If different inorganic composition is targeted, such as alumina or zirconia, their respective precursors should be added instead of the alkoxysilanes.

It is also possible to obtain these mesoporous materials following a different path, which is based in the coassembly between the inorganic precursor and the structure directing agent. To achieve this, both the precursors and surfactant should be added at the same time and the mesophase formation would take place at the same time than hydrolysis + condensation.

In any case, the final step of the synthetic process consists on the surfactant removal from the product to yield a network of cavities within the inorganic framework. The surfactant can be removed from the product through calcination at high temperatures or solvent extraction under softer conditions.

\section{Their Properties}

Since the discovery of silica mesoporous materials is back to the nineties $[1,2,14]$, many structurally different mesoporous materials have been produced through the templating method using the self-organization properties of amphiphilic under either acidic or basic conditions. In this sense, it is worth mentioning the use of block copolymers to produce ordered mesostructure silica with pores ranging from 5 to $30 \mathrm{~nm}$. The most famous material produced using those block copolymers is SBA 15, which was discovered in Santa Barbara, CA, back in 1998 [15]. Additionally, there are many research groups that have developed many other ordered mesoporous materials with different structures and, consequently, properties. 
As it has been mentioned above, ordered mesoporous materials are structurally unique solids with order on the mesoscopic scale and disorder on the atomic scale. The materials consist of channels, cages, or pores supported/separated by amorphous silica walls. Those cavities are arranged periodically on a lattice as artificial atoms or molecules in ordinary crystals, and this is why those materials are also known as "cavity crystal"

The synthetic conditions present a strong influence on the mesoscale order, which can show local structural variations. To characterize those structural properties and porous characteristics the use of electron crystallography has been vital. In this sense, X-ray diffraction (XRD) powder profiles of mesoporous materials normally show a few broad peaks, which make the structural characterization employing only XRD very difficult. Taking into account that electrons interact with materials much more strongly than X-ray and electron scattering amplitudes are approximately $10^{4}$ times larger than those of X-rays, it is possible to obtain the same structural information using electrons from a crystal volume $10^{-8}$ smaller than with X-rays. It is for this reason that crystals on the order of tens of $\mathrm{nm}$ are sufficiently large for single crystal transmission electron microscopy observation.

In this sense, two transmission electron microscopy images with incidences parallel and perpendicular to the channel present evidence of $p 6 \mathrm{~mm}$ symmetry and a monodimensional channel system. It was in 1992 when Kresge et al. proposed the combination of the electron microscopy observations with powder XRD results to solve the structure of the first mesoporous material discovered, MCM-41 [2]. This work clearly evidences the importance of TEM studies in the structural characterization of mesoporous materials. However, there are many publications in this area with an unfortunate mix of speculative structures and structure solutions. In this sense, the work carried out by Terasaki et al. is of a great value because it shows the way that structural characterization should be done [4-8]. His experience in structural characterization techniques helped him to combine high-resolution transmission electron microscopy with electron crystallography, leading to the solution of the structure of a considerable number of mesoporous materials. Thus, the exhaustive characterization work carried out by his research team was the key to settle the relationship between mesostructure and material properties, opening the gates to novel and exciting applications for those mesoporous materials.

Even though TEM analysis is a very powerful technique, it should be recalled that a TEM image essentially projected structural information of a determined specimen along the direction of the incident electrons. For this reason, to obtain $3 \mathrm{D}$ structures, it is necessary the combination of images from different incidences in terms of Radon transformation of Fourier transformation $[16,17]$. In this sense, the combination of thousands of projected images through "filtered back projection" allows the determination of the structure of $3 \mathrm{D}$ objects. This technique, called tomography, is widely employed in medical imaging and is also very useful to study determined nanostructured materials.
However, when the analyzed material is a crystalline per-iodic system, it is possible to employ crystallography rather than tomography. Thus, through the utilization of crystallography is possible to dramatically reduce the number of images required. By this way, the higher is the crystal symmetry the fewer is the number of images necessary. Additionally, it is also possible to reduce the $\mathrm{S} / \mathrm{N}$ ratio because all the information is concentrated only on reciprocal points. The information on the periodically averaged structure can be collected over the region of high-resolution transmission electron microscope micrograph where the Fourier diffractogram is obtained (Figure 1).

\section{Their Applications as Drug Delivery Systems}

As it has been mentioned above, ordered mesoporous materials were initially developed for catalysis applications. However, many researchers quickly recognized their potential for other applications in many different research areas, such as magnetism, sensors, optical materials, photo catalysis, fuel cells, thermo electrics, and even in the healthcare research area.

It was in 2001 when our research group proposed these ordered mesoporous materials as drug delivery systems for the first time [18]. Essentially, pharmaceutical agents were loaded into the mesoporous cavities to be then released as required in the body. Obviously, the lack of toxicity is a must for this type of application.

Since then, silica-based ordered mesoporous materials have experienced a growing interest by biotechnological researchers due to their potential to host very different guest molecules. An important point within this technology is the host-guest interaction that would take place between the silanol groups located at the surface of the host matrices and the functional groups from the guest molecules. This interaction would have a strong effect on the drug adsorption and release properties of the carrier matrices $[19,20]$. Additionally, textural and structural properties have been observed to modulate the adsorption and release characteristics of these ordered mesoporous materials [21].

When dealing with these ordered mesoporous materials for drug delivery, the first and perhaps most important condition is the correct selection of the mesoporous material from all those available depending on the molecule to be hosted [22]. In this sense, when targeting molecules with a size of few nanometers, MCM 41 silica materials seem to be the correct choice since they are stable, with pores of $c a$. $2-3 \mathrm{~nm}$, large surface area and very easy to functionalize to modulate the adsorption and release rates.

An important breakthrough in this technology supposed the possibility of visualizing the drug molecule inside the mesopores. Up to that moment, the combination of indirect characterization techniques was necessary to ensure the drug loading into the inner part of the mesopores. The use of aberration corrected high-resolution electron microscopy by Vallet-Regí et al. allowed to detect the drug molecules confined into the pore channels of silica-based ordered 

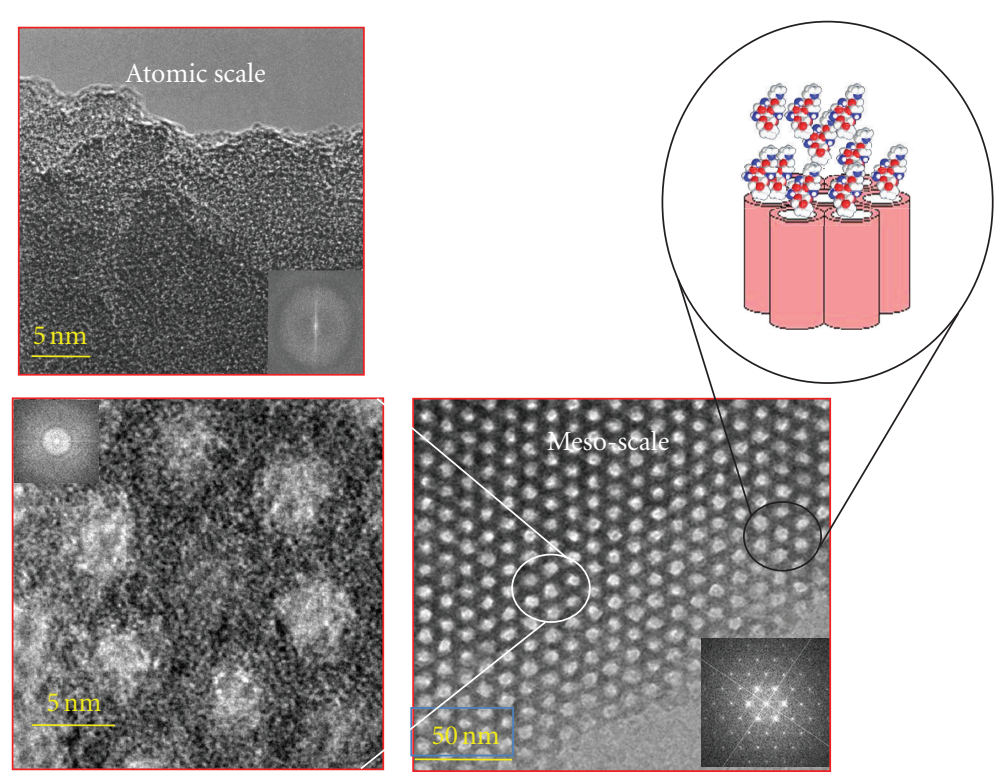

FIgURE 1: Transmission electron micrographs and Fourier transforms of conventional sol-gel glasses (top) and mesoporous or templated glasses (bottom).

mesoporous materials [23]. This work was possible using Cs correctors incorporated to a STEM microscope allowing the illumination of an individual atom with the electron beam to identify an unknown substance and, therefore, ensuring that the drug molecule was inside the mesopore channel (Figure 2).

However, to calculate the amount of adsorbed drug, it is necessary to combine different indirect methods such as porosity, specific surface area measurements, thermogravimetry and chemical analysis.

The release of the drug from the mesoporous matrix would take place through diffusion of the pharmaceutical molecule throughout the pore channels. However, when targeting a truly controlled release, it is necessary to design stimuli-responsive systems, where the drug is released under certain stimuli, such as $\mathrm{pH}$, temperature, ultrasounds, or light.

An important feature of these silica-based ordered mesoporous materials is the possibility of organically modifying their surface through the covalent attachment of many different functional groups [24]. As a consequence of the functionalization, organic-inorganic hybrid materials are obtained, which can adsorb many different drug molecules through weak interactions.

The functionalisation of the silica walls may be necessary for several reasons. In some cases, there are certain drugs with remarkable hydrophobic nature that do not exhibit any trend to penetrate into the hydrophilic mesoporous silica [25]. The functionalisation with hydrophobic functional groups is a good alternative to promote the load of different hydrophobic drugs. This strategy is also employed to delay the release kinetics of certain drugs from mesoporous channels to the aqueous release medium due to the decrease in the wettability degree of the material surface [26].
On the other hand, there are other situations in which the pharmaceutical molecule can be confined into the mesoporous channels. However, higher loads and slower release kinetics can be achieved if the mesoporous silica wall is functionalised with different functional groups. Among the existing organic groups, the functionalisation with aminomoieties has been widely reported [27].

One of the great advantages of these ordered mesoporous materials is the great versatility of the synthetic process, which allows the production in bulk but also as microcapsules and even as nanoparticles.

Certainly, it was far from their thoughts (from Kuroda and Kresge teams) that their discovery, devoted to catalytic applications, could be later on an excellent tool in medicine and nanomedicine. Actually, magnetic nanoparticles have been encapsulated into inorganic mesoporous nanoparticles of silica for applications against cancer, able to design smart nanoparticles which besides are able to simultaneous and sinergically perform a double function, to delivery in a controlled way citotoxics and to give off heat (hyperthermia). The stimuli responsive effect can be applied for on-demand release of the drug [28].

The presence of silanol groups on the surface of silica facilitates the reaction with alcohols and organosilanes, is able to stabilize the suspensions in nonaqueous media, as well as provides groups for the attachment of specific ligands. Gene transport and delivery by magnetic conjugates represents a particular challenge. DNA fragments have to overcome several biological barriers when reaching the cell nucleus from the extracellular environment [29].

The covalent bonding of dendrimer molecules to magnetic nanoparticles has been revealed as a promising strategy to perform magnetic force-assisted transfection in vitro. In this kind of systems, the magnetic component is aimed at 

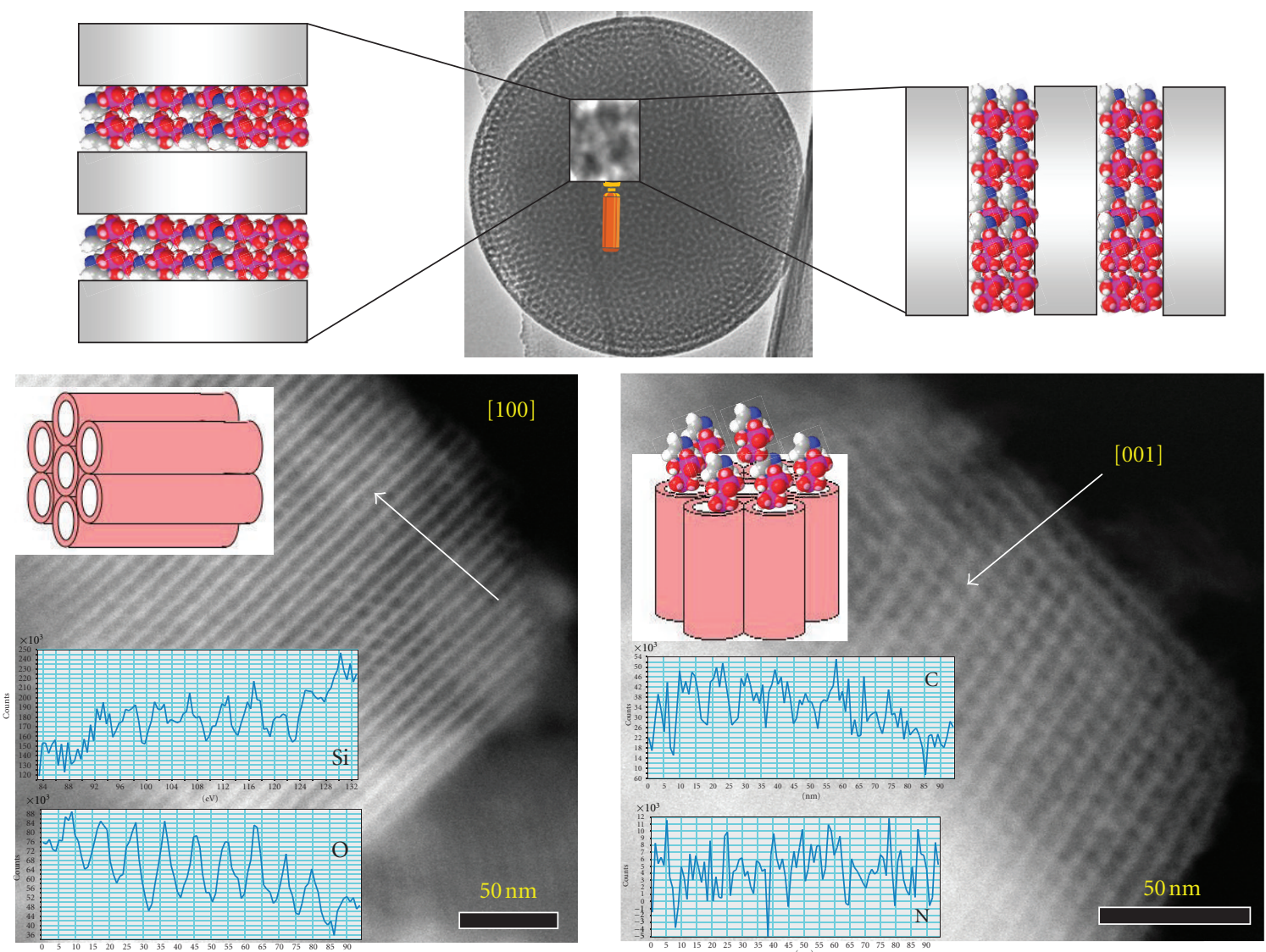

Figure 2: Transmission electron micrographs of silica mesoporous particle loaded with zoledronate with a schematic representation (top) and Cs correctors incorporated to a STEM microscope to directly visualize the zoledronate loading (bottom).

facilitating an intimate contact between cell culture and gene vectors, thus providing a reduction of transfection times. Many of the systems proposed in the literature as selective drug carriers are based on core-shell settings in which various species, as is the case of silica mesoporous nanoparticles.

The development of novel advanced multifunctional materials for a broad range of technological applications represents a renovated hope in many different fields. In particular, recent research breakthroughs in the biomedical arena have emerged as the basis for future personalized treatments and diagnostic techniques with a hitherto unsuspected selectivity.

With the amazing advances in the preparation and characterization techniques of nanotechnology products, the possibility of manufacturing devices capable of establishing an intimate interaction with the biological world has been opened.This fact represents a precise control over the processes of therapeutic substances release and means an opportunity to improve the specificity of the therapeutic action, as well as to reconsider some of the promising drugs for certain diseases that were once discarded by their low levels of tolerance as discussed elsewhere.

The targeting ability of these new nanodevices should lead to tailor-made dosing regimens, with a significant reduction of severe side effects associated to some diseases, such as cancer, and should also eventually result in a more efficient allocation of health care resources. Moreover, the use of these engineered products may allow the combination of the therapeutic potential and nanoscale diagnosis with low tissue invasiveness. And in many of these cases, silica mesoporous nanoparticles are being used as a consequence of the discovery at the early nineties, even though their discoverers were probably unaware of this application since they were involved in the world of catalysts. But, anyway, they were able to establish the basis to develop new synthetic pathways to be used in many different research areas and, among them, the medical field.

Right now, Chemistry Science offers many different possibilities to produce silica mesoporous materials, which means between two and fifty nanometers of porosity. Although the initial synthesis was carried out in bulk materials, nowadays is possible to produce mesoporous nanoparticles with a similar composition [30].

As it has been commented above, in the specific field of silica mesoporous materials we can induce weak interactions between this ceramic matrix and different drugs in order to obtain efficient controlled drug delivery systems. On the other hand, if we induce strong interactions between the organic matrix and biologically active molecules that enable bone tissue regeneration, we will produce efficient 
systems for hard-tissue regeneration. Simultaneously, we can combine weak and strong interactions in the same system, which would then be suitable for both purposes: drug delivery and tissue engineering, which is an important characteristic of this type of materials [25].

Concerning hybrid bioceramics the combination of both inorganic and organic constituents produces a remarkable synergy that makes them appropriate for diverse medical applications. The weak interaction of hybrid bioceramics with drug molecules hosted into the mesoporous cavities permits the design of controlled delivery systems. In addition, mesoporous silicas can be used as starting materials for the manufacture of three-dimensional (3D) scaffolds for bone tissue engineering [20, 30-33]. To attain satisfactory biological response, different osteoinductive molecules, such as peptides, hormones, and growth factors, should be strongly attached to the bioceramic surface, acting as signaling agents for cells to assist bone regeneration process $[30,34]$. In addition, there are many research examples regarding hybrid bioceramics as stimuli-responsive drug delivery systems and nanosystems for targeting of cancer cells and gene transfection $[11,25,29,35-42]$.

Multifunctional nanoplatforms suitable for nanomedicine applications must satisfy major requisites; the biocompatibility of the nanocarrier material is a mandatory issue; the nanocarriers must provide high loading and safeguard capability of desired therapeutic or imaging agents; the nanosystem must avoid premature departure of entrapped drugs before reaching its target, meanwhile exhibiting cell type or tissue specificity and site targeting capability, efficient cellular uptake, effective endosomal escape, and tunable delivery rate to accomplish effective local concentration.

Multifunctional nanosystems that have been widely investigated for application in nanomedicine are either organic or inorganic materials (Figure 3). Among organic nanoparticles we can find dendrimers [43], liposomes [44, $45]$, polymers [46, 47], and virus-like particles [48].

Inorganic nanosystems have been also widely explored, including gold nanoparticles [49], semiconductor nanocrystals [50], superparamagnetic nanoparticles [51], and silicon [52] and silica-based nanoparticles [53, 54]. Currently, inorganic nanoparticles are gaining increasing significance since they exhibit higher mechanical strength, chemical stability, biocompatibility, and resistance to microbial attack than their organic analogous $[55,56]$. Moreover, the inorganic matrix efficiently helps preserving the chemical nature of entrapped molecules by protecting them from degradation or denaturation provoked by enzymatic attack or by $\mathrm{pH}$ or temperature variations in the local environment.

Within the context of inorganic nanomaterials, mesoporous silica nanoparticles (MSNPs) have arisen as hopeful multifunctional platforms for nanomedicine (Figure 4). Since mesoporous materials burst on drug delivery stage in 2001 [57], MSNPs are receiving increasing research attention for their forthcoming applications in biotechnology and nanomedicine $[11,25,29,35-37,39,41,42,58,59]$.

MSNPs suitable for nanomedicine applications require synthesis methods that produce uniform nanoparticle sizes in the $30-300 \mathrm{~nm}$ range and minimize self-aggregation of nanoparticles. Accordingly, the two main strategies used to synthesize MSNPs are the so-called "modified Stöber method" and the "aerosol-assisted synthesis". The former consists in the silica condensation under basic medium using cationic surfactants as template agents. Aggregation of nanoparticles is reduced by the alkaline and highly diluted conditions, which favour negatively charged and more fullycondensed surface [60]. Aerosol-assisted synthesis permits synthesizing MSNPs by using not only cationic but also anionic and even nonionic surfactants under basic or acid conditions [61-65]. Whatever the followed synthetic approach, the last stage consists in the surfactant elimination, which usually leads to materials with cylindrical mesopores organised in a two-dimensional hexagonal way, typical of MCM-41-type materials [2]. MSNPs present singular and valuable textural and structural characteristics, such as high surface area $\left(c a .1000 \mathrm{~m}^{2} / \mathrm{g}\right)$ and pore volume $\left(c a .1 \mathrm{~cm}^{3} / \mathrm{g}\right)$, stable mesostructure, narrow pore size distributions that can be tuned in the $2-10 \mathrm{~nm}$ range, two functional surfaces (outer particle and inner pore faces), and tuneable particle size. When aiming at targeted intracellular delivery the MSNPs size must be within the 50-300 nm range. The synthesis of MSNPs with sizes smaller than $50 \mathrm{~nm}$ is not easily achievable due to the inherent mesoporosity of nanoparticles. On the other hand, nanoparticles larger than $300 \mathrm{~nm}$ cannot easily cross physical membranes in the body. The particle size has been proved to be one of the key factors that dictate the final fate of nanoparticles in living systems [66-68]. For this reason, narrow particle size distributions are desired and therefore, wet-chemical synthesis routes are normally preferred to other physical methods such as spraydrying, which normally leads to somewhat broad particle size distributions.

The presence of high density of surface silanol groups capable of being functionalized using a wide variety of entities represents one of the great milestones of MSNPs $[24,69,70]$. When nanomedicine applications are aimed, the surface functional groups of MSNPs can play diverse roles, such as tailoring the surface charge of nanoparticles, allowing the grafting of functional molecules inside and outside the pores or facilitating the capping of the nanopore openings to prevent premature release of entrapped drugs.

Functionalization of MSNPs is mainly achieved using three distinctive methods: cocondensation, postsynthesis and surfactant displacement. The cocondensation method involves the synthesis and functionalization in a unique step, for instance the organosilanes are added during the synthesis stage together with the silica source $[24,61,63,69,70]$. The surfactant removal is carried out by ion exchange using either an ethanolic solution of ammonium nitrate [71] or hydrochloric acid $[72,73]$. This easy synthetic procedure leads to MSNPs displaying uniform distribution of functional groups. Nonetheless, sometimes, depending on the used solvent, the surfactant extraction is incomplete, which can represent a troublesome for the further nanomedicine applications of the resulting nanoparticles. The postsynthesis or grafting method consists in grafting the functional groups after the surfactant removal, either by extraction or 


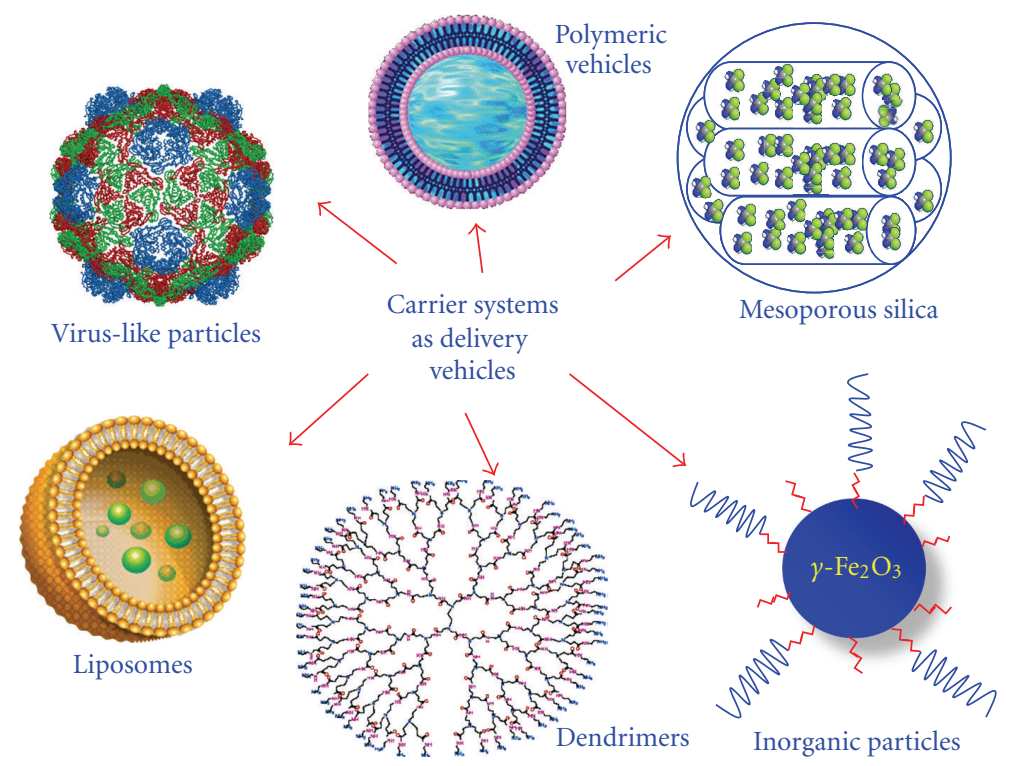

FIgURe 3: Different carrier systems used as release vehicles for smart drug delivery.

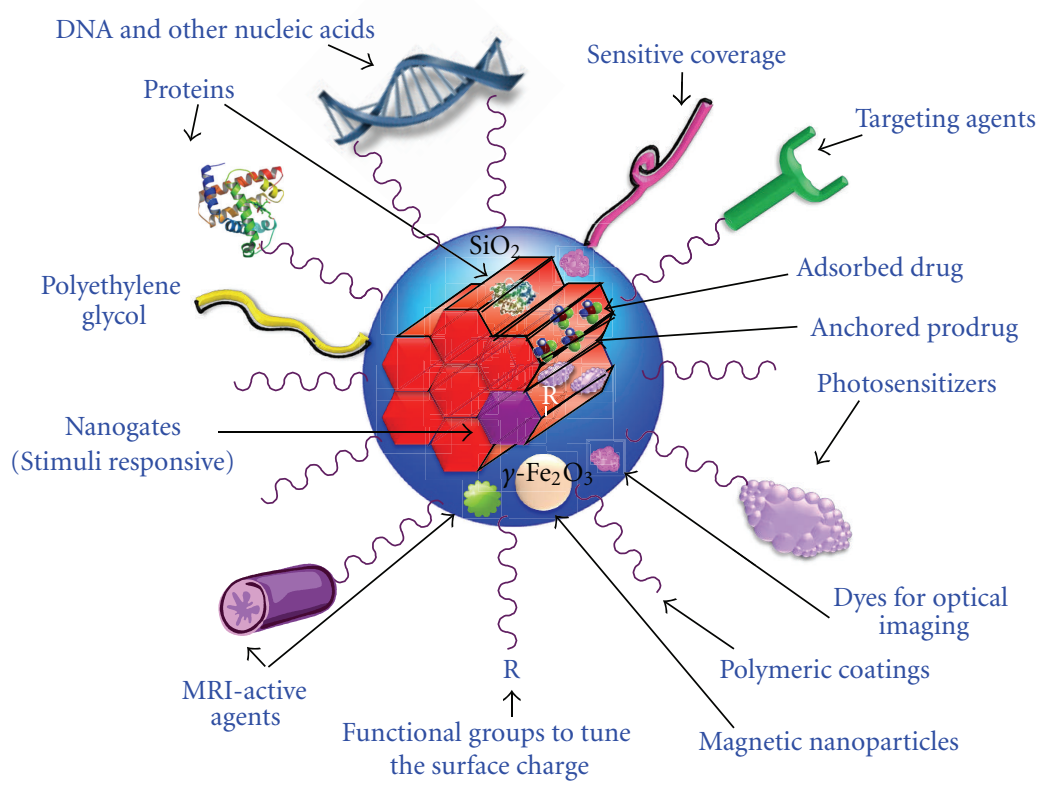

FIGURE 4: Mesoporous silica nanoparticles as nanomedical multifunctional nanoplatforms.

calcination, has been carried out [74]. This method offers a wide range of chances to allocate diverse functional groups in MSNPs and also allows to chemically attach delicate organic functions prone to hydrolysis and elimination reactions [75]. One of the major issues of postsynthesis method is the possible blocking of the mesopore openings by the functional groups, which would lead unavoidably to heterogeneous functionalization of the mesoporous matrix [76]. Last, the surfactant displacement method consists in the direct surface silylation with simultaneous surfactant removal by using acidic alcohol as solvent $[77,78]$. This method produces homogeneous monolayer coating, where the amount of functional groups on the mesoporous silica surface can be precisely tuned.

It should be highlighted that the exclusive topology of MSNPs allows distinguishing three well-defined domains that can be independently functionalized: the silica framework, the mesopores, and the outermost surface of nanoparticles. Owing to the remarkable characteristics of MSNPs they are excellent nanoplatforms to integrate several multifunctionalities for therapy and diagnostic of different pathologies [11, 29, 37, 41, 58, 59]. 
Therefore, different molecules can be incorporated into the silica matrix acting as contrast agents for optical imaging (OI) and magnetic resonance imaging (MRI). It is also feasible to entrap magnetic nanoparticles within the silica framework capable to play a dual role; contrast agents for MRI and thermoseeds for the treatment of tumors by hyperthermia. In addition, mesoporous channels can accommodate diverse organic molecules, such as drugs, proteins, nucleic acids for gene therapy, or photosensitizers for photodynamic therapy (PDT). These molecules can be simply adsorbed or covalently grafted to the inner part of the mesopore walls. A ground-breaking approach consists in providing MSNPs of stimuli-responsive controlled delivery capability. To tackle this goal, molecular nanogates can be covalently anchored to the pore outlets, obstructing the mesopore entrances and therefore avoiding premature departure of entrapped cargo. A given stimulus would produce the aperture of the nanogates and trigger the release of molecules in the target cell or tissue. To optimize the performance of MSNPs for diverse nanomedicine applications, the outmost surface of nanoparticles with targeting agents for specific cellular uptake, functional groups to modulate surface charge, polymeric coatings such as poliethyleneglycol (PEG) to provide the system of "stealth" properties or stimuli-responsive polymers, MRI contrast agents, photosensitizers, nucleic acids, proteins, and so forth. The high versatility of MSNPs permits designing multifunctional nanoplatforms for "theranostic", that is. therapy and diagnostic, nanomedicine.

\section{Some Examples of Mesoporous Nano and Micro particles for Drug Delivery Systems}

It is possible to synthesise nano and microparticles by pyrosol method, for example, calcium and phosphorous mesoporous matrices [61]. And it is possible to include magnetite, for instance. These magnetic nanoparticles can also be integrated in drug delivery systems. And they can be loaded with drugs and perform a controlled release [79].

Bioactive microspheres can be prepared following the evaporation-induced self-assembly (EISA) method. With this strategy, the ordered mesoporous structure can be kept even in multicomponent systems such as $\mathrm{SiO}_{2}-\mathrm{CaO}-$ $\mathrm{P}_{2} \mathrm{O}_{5}$. The mesoporous order is closely related with the structure directing agent as well as its interaction with the $\mathrm{Ca}^{2+}$ cations during the mesophase formation. For instance, the triblock copolymer F127 leads to hexagonal ordered structures for low $\mathrm{CaO}$ contents, P123 leads to wormlike mesoporous structures for any $\mathrm{CaO}$ content, whereas the ionic surfactant cetyltrimethyl ammonium bromide $\left(\mathrm{C}_{16} \mathrm{TAB}\right)$ does not produce accessible mesopores at the external surface, for any $\mathrm{CaO}$ content. Initial studies have demonstrated that this microparticles have the capability to load and release triclosan. In this sense, the kinetic profiles for drug release strongly depend on the pore structure, thus showing interesting features to be used in periodontal regenerative surgery and infection prophylaxis [61].

It is also possible to design silica mesoporous particles. Which can be also synthesised with magnetic nanoparticles.
Following the same strategy of aerosol-assisted methods, silica mesoporous spheres containing magnetic nanoparticles can be also synthesized. In this case, not only the kind an concentration of surfactant but also the concentration of magnetic nanoparticles affect the mesoscopic ordering degree of the materials. Magnetic nanoparticles must be incorporated to the precursors solution. However, after the EISA process, they do not undergo neither crystallinity changes nor superparamagnetic loss in the encapsulation process. In addition, the silica shell of these materials provides an appropriate surface to achieve stability at physiological $\mathrm{pH}$. Mesoporous magnetic microspheres are a nice example of multifunctional material. The combined properties of magnetic nanoparticles and silica microspheres make these materials excellent candidates for biomedical applications that would involve hyperthermia treatments and drug delivery.

These particles loaded with drugs and magnetic nanoparticles inside can be used in hyperthermia treatments, controlling time and temperature, in order to eliminate carcinogen cells or treated as a drug delivery system, with the adequate cytotoxic products to destroy carcinogen tissue [80].

The properties and broad range of applications of colloids formed by uniform sized nanocrystals have awaken a special attention in several research areas [81, 82]. Among them, the capability for information storage, catalysis and biomedical uses of magnetic nanoparticles has attracted the attention of the scientific community [83-85]. Magnetic nanoparticles have been proposed for different applications in the field of diagnostics and clinical therapy. Thus, they are currently used as contrast agents for magnetic resonance imaging, whereas their potential applications as carriers for drug or gene delivery systems, DNA and other biomolecules separation, and hyperthermal treatment of tumours have been also consideredas [86-88].

The use of magnetic biomaterials for drug delivery is a very interesting strategy for specific drug targeting and release. Actually, magnetic nanovehicles provide the ability to be directed and concentrated within the target tissue by means of an external magnetic field and to be removed when the therapy is completed. For this purpose, it is highly desirable that magnetic nanoparticles were superparamagnetic, that is. they do not keep magnetized after the action of the magnetic field, thus reducing the risk of particle aggregation. Another capability of superparamagnetic nanoparticles, is the heating power that exhibits when they are exposed under an alternating magnetic field. This fact is the result of Brownian rotation and Neel relaxation mechanisms, thus allowing to reach local hyperthermia temperatures for cancer treatment $[89,90]$.

For biomedical purposes, the most studied magnetic nanomaterials are those based on iron oxide magnetic nanoparticles, either magnetite or maghemite (Figure 5). This fact can be understood in terms of their behavior due to their chemical stability and biocompatibility when compared to metallic nanoparticles [91]. Anyway, the functionalization with a suitable coating is desirable in order to protect the material against the aggressive environment inside the body. In addition, this coating should provide enough surface 

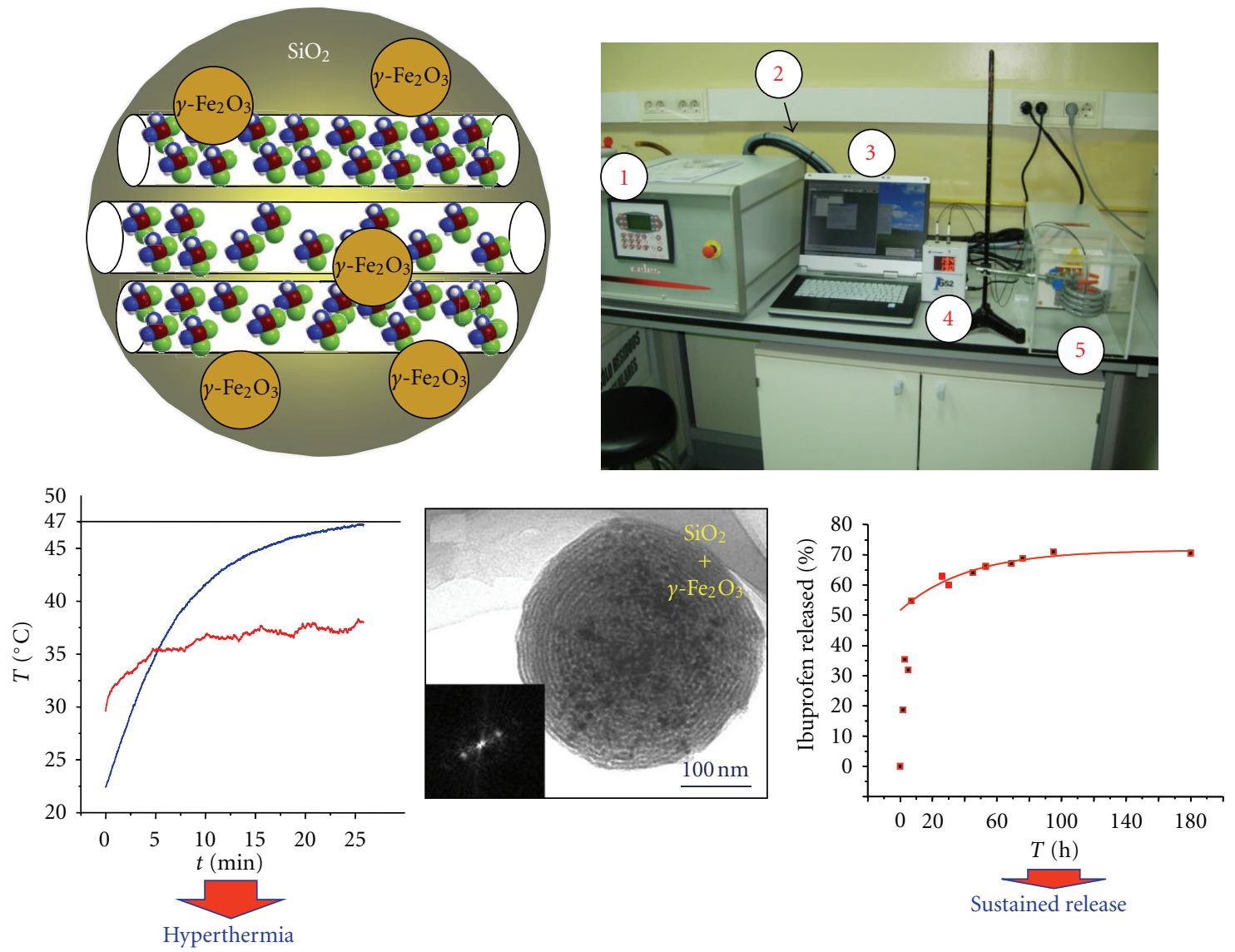

Figure 5: Schematic representation of MSNPs encapsulating $\gamma$ - $\mathrm{Fe}_{2} \mathrm{O}_{3}$ nanoparticles and loaded with drugs. Transmission electron microscopy (TEM) image of the system and its corresponding Fourier Transform are also displayed. Hyperthermia equipment, controlling time and temperature and drug delivery.

electrical repulsion to avoid their aggregation under the magnetic field. In this sense, organic and inorganic shells have been investigated $[92,93]$ and the magnetic properties of the material, its stability under physiological conditions, and the potential for drug targeting and delivery are considered [94]. Among the inorganic coatings, silica appear as an appealing approach since the high density of silanol groups on the surface renders the magnetic material reactive to a variety of coupling agents [21]. As mentioned above, silica species can be organised by surfactants acting as structure-directing agents to produce well-ordered mesoporous networks. This is an excellent chance to prepare coatings with ordered porosity $[2,3]$.

Considering that mesoporous structures have demonstrated high drug loading capacities and controlled release kinetics [18, 22, 95-97], superparamagnetic iron oxide nanoparticles have been incorporated into mesoporous silica spheres. For this purpose, the EISA method has been employed, as explained above $[63,98]$.

It is also possible to produce spherical mesoporous particles of mixed oxides employing an aerosol for the synthesis. This approach is very interesting since it allows the synthesis of materials with a wide range of metal oxide combinations, which could be of interest to increase the adsorption and delay the release of certain bisphosphonate drugs [64]. In this sense, mesoporous silica-zirconia-mixed oxides were produced to be employed as delivery vectors of bisphosphonate-based drugs (Figure 6). These materials produced through the spray-drying method showed tunable acidity. To evaluate their capability as drug delivery systems, alendronate and zoledronate drugs were selected. They present a similar phosphonate head but different amine tail, which would lead to different acidity and hydrophobicity. The synthetic route employed aerosol allows the incorporation of different quantities of zirconium amounts into the mesostructured silica, which provoked the creation of different Lewis and Brönsted acid centers. Thus, the surface properties of the mesoporous matrix could be tuned, which allowed modulation of the bisphosphonate drug adsorption and release. Thus, these mixed ordered mesoporous oxides are demonstrated to be versatile controlled release systems of bisphosphonate drugs with potential application in bone implant biotechnologies.

In many cases, it is necessary to functionalise the surface of these particles with specific targeting agents to promote cell recognition and internalization to specific cells. 

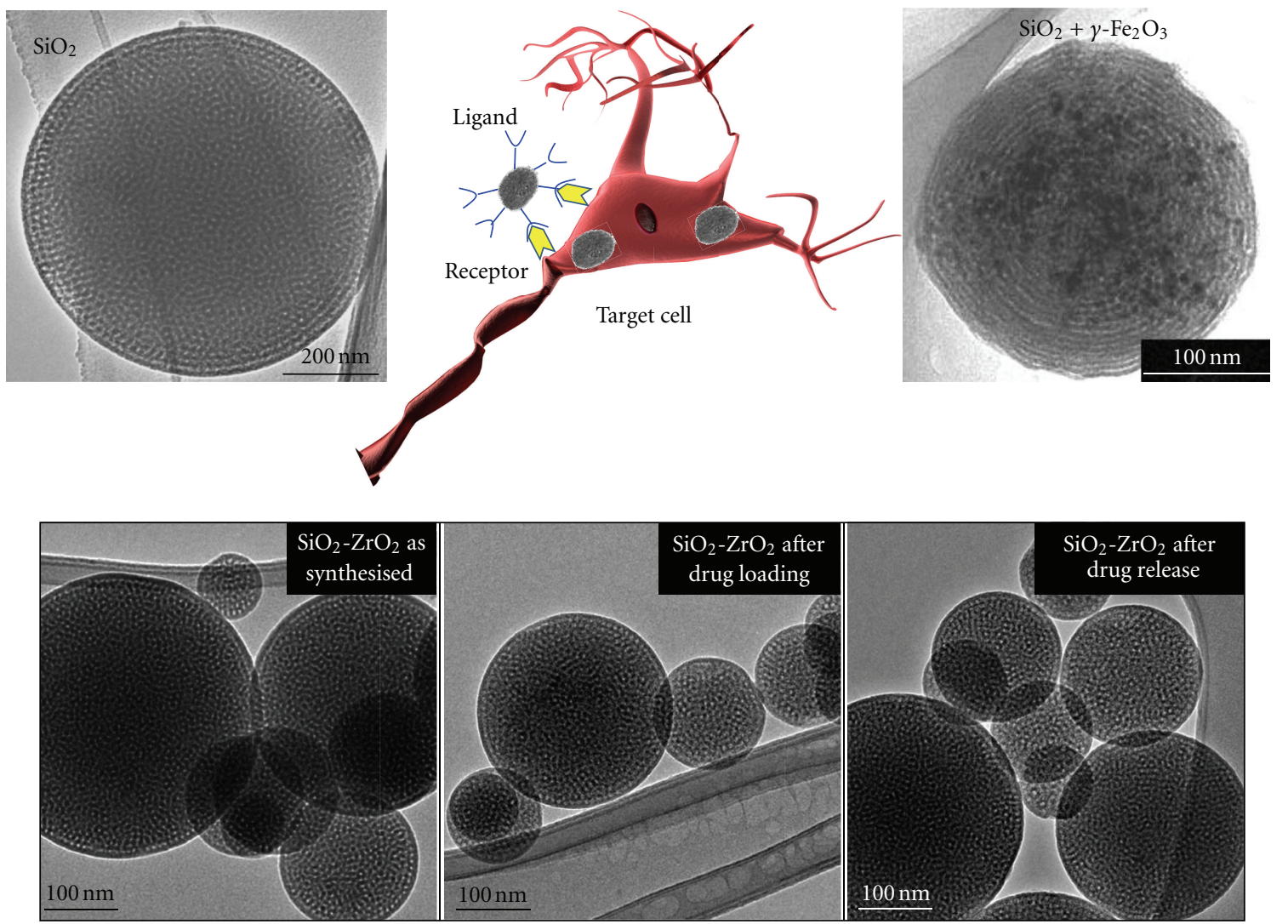

FIGURE 6: Mesoporous nanoparticles with different compositions; schematic representation of the targeting approach.

6.1. Stimuli-Responsive Materials for Drug Delivery Applications. The use of different external or internal stimuli such as $\mathrm{pH}$, temperature, ultrasounds, and light among others has been employed in order to achieve a controlled release of the drugs trapped inside mesoporous silica nanoparticles. Different aspects should be considered for the development of an effective stimuli-responsive system, such as the type of mesoporous matrix, the drug to be released, environment of the target tissue and specially, how to retain the drug until reaching the target zone and also releaing only the required dose (Figure 7 ). The combination of supramolecular chemistry with material science may open up excellent opportunities to achieve this goal. In 2001, the Vallet-Regí research group described for the first time that it was possible to load and release different drugs from a mesoporous silica matrix [18]. Moreover, these materials could host many different molecules. The performance of the silica nanocarriers was monitored employing a reporter molecule. This molecule should present a convenient size that allow it to enter and exit through the pore, an adequate electrostatic charge and solubility in the environment (usually aqueous solutions) and finally, the molecule should be easily detected (e.g., fluorescent molecules). The next step was to achieve a controlled release of the trapped drugs in such a way that only happened in the presence of certain stimuli. The system has to be able to transport a cargo to specific places in the body without premature leak during the voyage and release the payload by the application of the trigger stimuli once reached the target zone. Other important property in order to design an effective nanodevice for on-demand drug delivery is switchability [99] that means the capacity to be repeatedly activated by the trigger stimuli after reaching the targeted cells or tissues.

In many clinical situations, the administration of therapeutic compounds is hampered by impaired drug absorption or tissue-unspecific delivery [100, 101]. Moreover, in clinical fields as oncology, the high toxicity associated with the currently employed cytotoxic drugs converts the ratio risk-benefit unmanageable. In this particular field, the development of nano- and microparticles able to deliver different therapeutic agents and release them in a controlled manner in the affected tissues has emerged as one of the most groundbreaking application in nanomedicine [102105]. Within these novel carriers, magnetic nanoparticles are very promising candidates for active drug delivery systems due to their interesting properties. They can be concentrated and held in position by means of an external magnetic field [106] and their superparamagnetic behaviour provides the controlled heating capability necessary for the treatment of cancer by hyperthermia [107-111]. Moreover, these magnetic nanoparticles can be used for in vitro applications in macromolecule isolation (antibodies, proteins, etc.), cell separation, and detoxification of biological fluids $[87,112-114]$. As has been mentioned the 


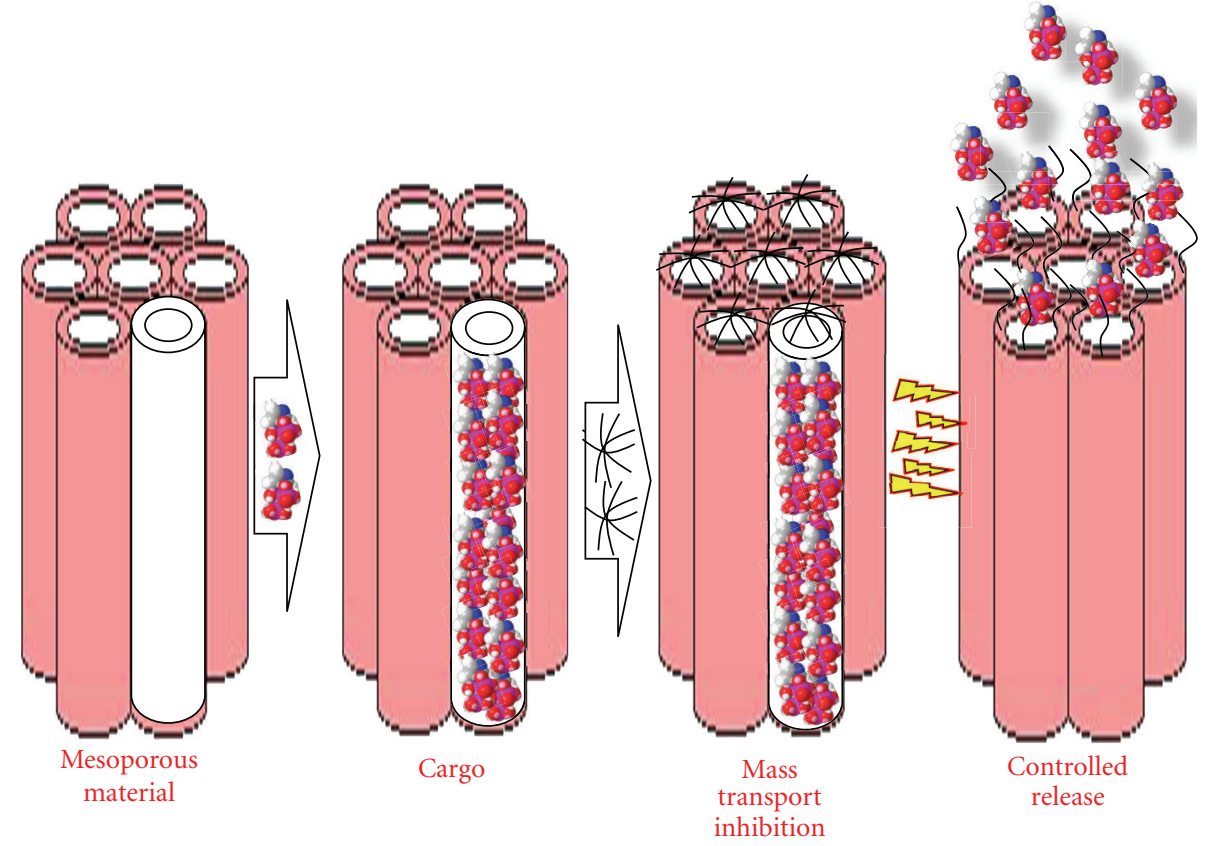

Figure 7: Schematic representation of the loading and control release in a smart mesoporous material.

previous section, mesoporous silica nanoparticles present very interesting properties for drug delivery applications, such as easy functionalization [115], good biocompatibility [116], stable mesoporous architecture, large surface areas, and tunable pore sizes and volumes, converting them in excellent candidates for controlled delivery of drugs $[18,21,95,117]$ or even macromolecules [118120].

In the recent years, an intensive work has been performed in the functionalization of the pore outlet of these materials with organic and bioorganic moieties as gating devices, able to control the release of the trapped molecules in response to different stimuli such as chemicals [121-125], $\mathrm{pH}$ [126-128], temperature [129], redox reactions [130132], enzymes [133], light [134-140], and antibodies [141] (Figure 8).

The use of magnetic mesoporous silica nanoparticles can be used in order to control the localization of the nanocarriers by an external magnetic field [142]. In our research group, alternative magnetic fields have been recently employed as trigger stimulus for the controlled release of a model drug (fluorescein) [143]. In this case, the ability of DNA chains to reversibly hybridize in response to temperature changes has been used as gatekeeping mechanism. This temperature-responsive behavior can be tuned through changes in chain length, variations in G/C content, and grafting density on the materials surface [144]. Thus, a previously fluorescein-loaded magnetic mesoporous silica nanoparticles were functionalized with 15 base pairs oligonucleotide single strands carefully designed to present a melting temperature with its complementary strand in the hyperthermia range $\left(45^{\circ} \mathrm{C}\right)$. Then, the system was capped with superparamagnetic iron oxide nanocrystals which carried the complementary oligonucleotide sequence, avoiding the premature release of the fluorescein trapped into the silica matrix. The application of the external alternative magnetic field caused the dehybridization between the two oligonucleotide strands due to the temperature increase allowing the release of the fluorescein molecules housed within the silica particles (Figure 9). The fluorescein release could be stopped once removed the magnetic field because the hybridization between the complementary strands was taking place again once the temperature fell below the melting value, which showed the on-off behavior of the nanodevice.

6.2. Mesoporous Motor Particle. The production of manmade nanomachines able to perform useful works employing free energy present in the environment is one of the greatest challenges in nanotechnology [145-147]. Nature is a inexhaustible inspiration source and produces some of the most complex motors known [148]. However, despite the fact that several groups have published the ability of harvested biomotors to perform different tasks outside the cell, short lifetimes associated to these complex biomolecules strongly limit its application [149-151]. As an alternative to the natural motors, the catalytic decomposition of hydrogen peroxide to oxygen and water has been widely used as energy source by many of the described systems able to perform translational and rotational non-Brownian motion [152-161]. Metallic nanorods have been employed in order to control the directionality of the motor and also to allow the transport and release of magnetic loads [162, 

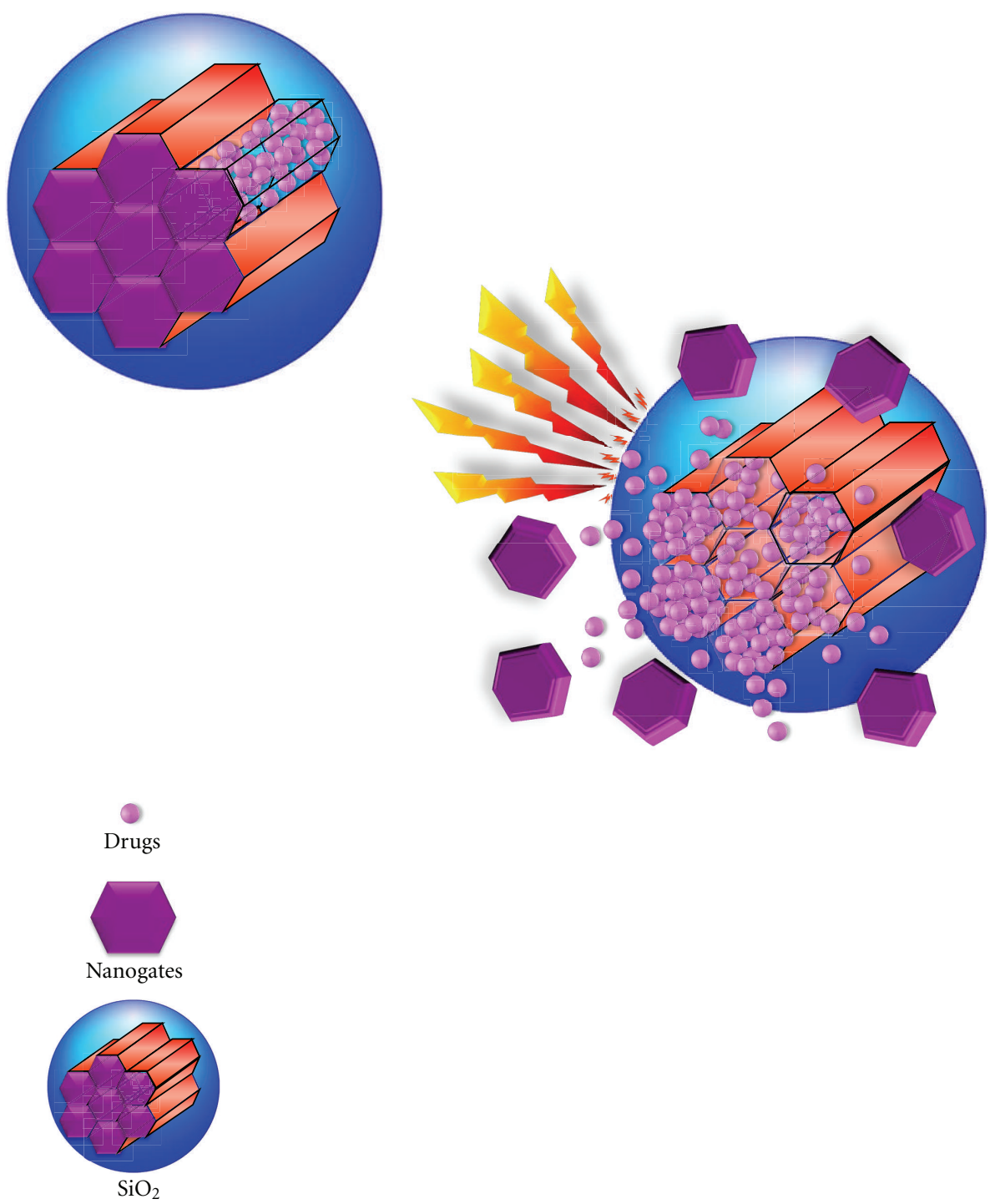

FIGURE 8: Schematic representation of stimuli-responsive drug delivery from silica-based ordered mesoporous materials. The different stimuli that have been used to trigger drug delivery include $\mathrm{pH}$, temperature, redox potential, light, magnetic field, ultrasounds, enzymes, antibodies, aptamer targets, or even the combination of more than one stimulus.

163]. Recently, rolled-out microtubular devices have been introduced in the motor arena. These microrockets are able to pick up and transport different cargos showing high propulsion rates and cargo capacity [164-166]. Moreover, it is possible to control the directionality of the motion through the application of an external magnetic field [167]. The use of nanomotors for medical applications required energy sources compatible with biological environments (Figure 10). Different strategies have been recently described such as fuel-free nanosystems able to work under electric [168] or magnetic fields $[169,170]$. Other interesting strategy is the integration of biomolecules on the surface of the artificial engines in order to perform the useful work exploiting the chemical energy present in the media. Thus, catalase has been anchored on metallic nanorods [171] and also on the internal side of the rolled-up gold microtubes [172] allowing the motion of these devices in low-concentrated hydrogen peroxide solutions that are a less aggressive media for clinical diagnostic applications. Enzymatic tandem reactions that employ glucose oxidase and catalase [173] or bilirubin oxidase [174] have been used as propulsion source of micrometer or millimeter objects through the catalytic decomposition of glucose present in the media, which could constitute a very promising approach for the use of these devices within living organism. Practical applications of these propelled nano- and microdeviecs are just emerging. For instance, these microshuttles have been employed for transport and release of drug-loaded nanoparticles [175], capture and transport of cancer cells or nucleic acids [176, 177] and finally, quantitative detection of different analytes such as silver ions or specific oligonucleotide sequences [178, 179].

In our research group, an innovative self-propelled nanodevice based on Janus mesoporous silica particles able 


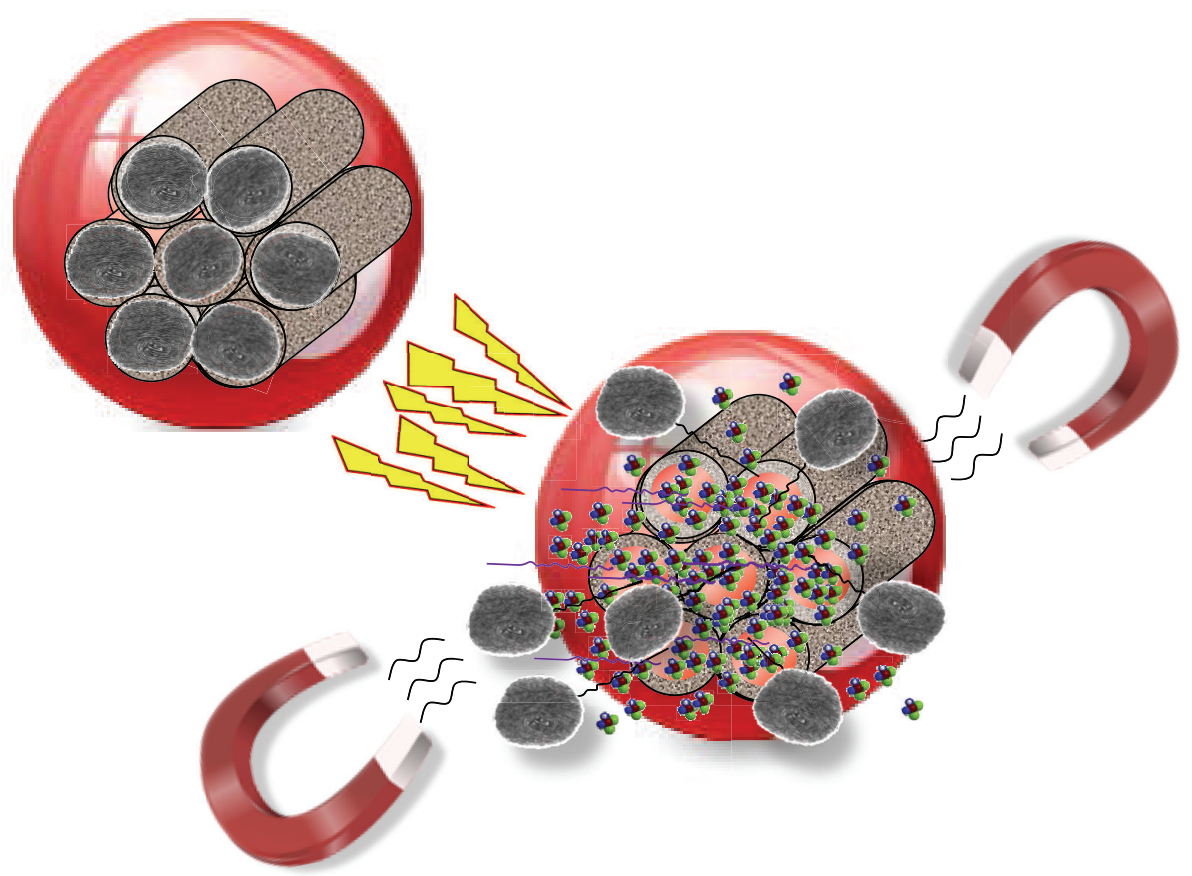

FIGURE 9: Schematic representation of MSNPs as stimuli-responsive drug delivery devices. Firstly drug is loaded into the mesopore channels. The pore openings are then closed using nanocaps to prevent premature departure of cargo. Finally, the application of a stimulus provokes the removing of the gatekeepers allowing the release of the entrapped drug.

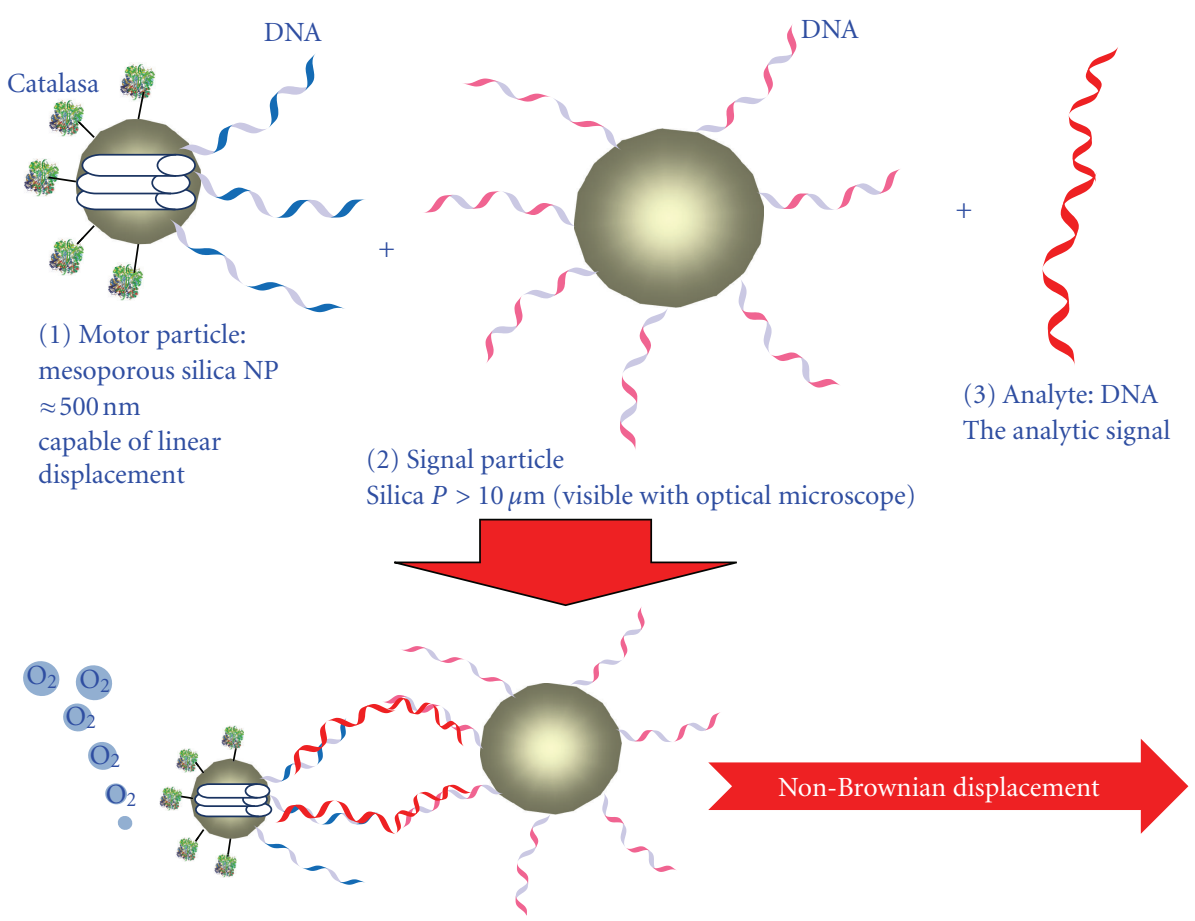

FIGURE 10: Three components to fabricate a mesoporous nanomotor.

to perform motion, cargo transport, and target recognition has been recently described [180].

These nanoparticiples constitute a very interesting alternative to already existing applications not only by their easy functionalization but also by the possibility to transport molecules trapped within the silica matrix. In this case, silica particles were asymmetrically functionalized with catalase and a specific oligonucleotide sequence in each side. The enzyme provides the swimming capacity through the generation of oxygen bubbles in a low-concentrated 


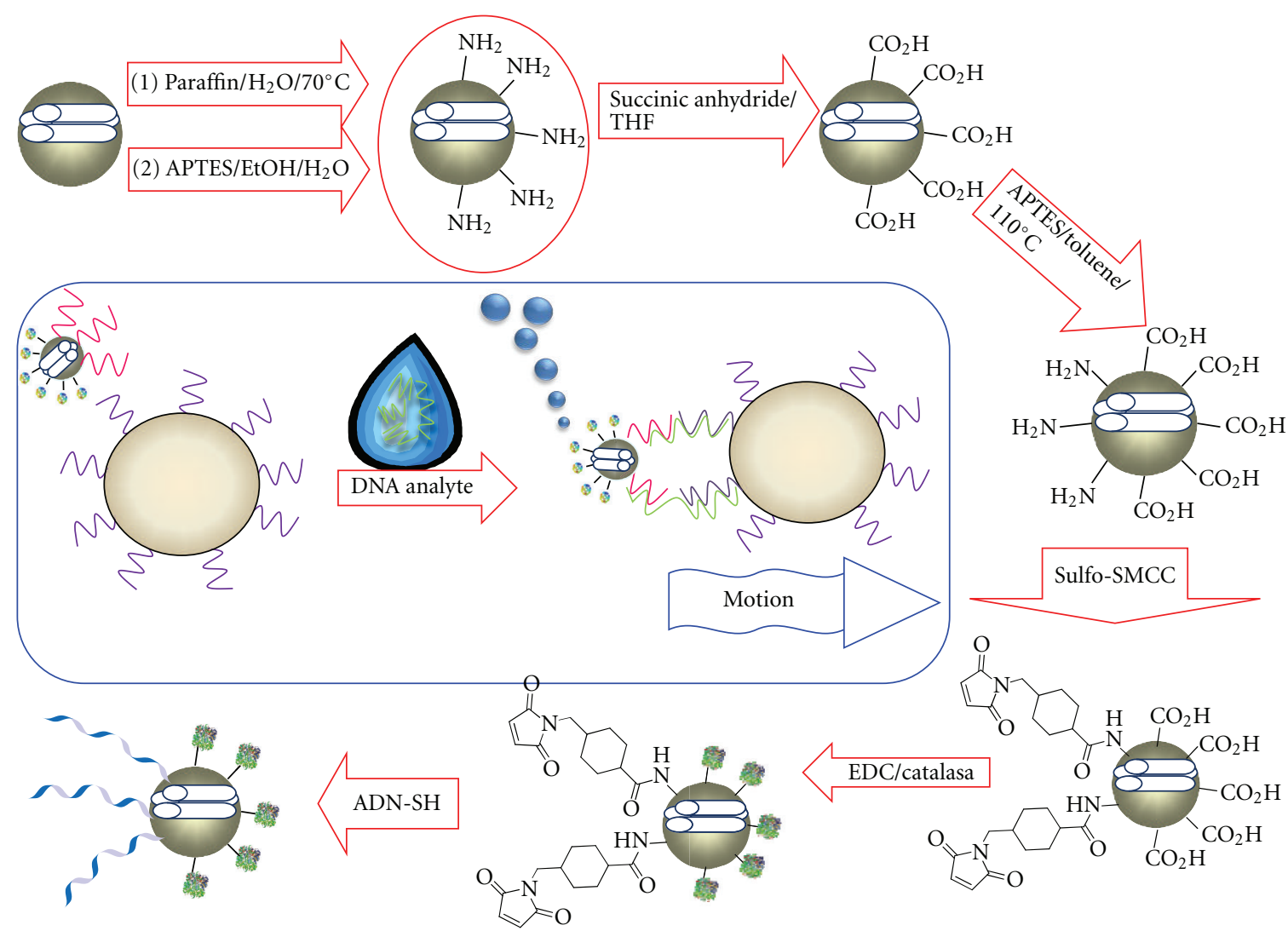

FIGURE 11: Scheme of the synthesis of nanoparticle motor. In the center representation of the motor mechanism.

hydrogen peroxide solution. The oligonucleotide sequence attached on the opposite face allows the selective capture of a signal particle, also functionalized with other DNA sequence. Both DNA strands are not complementary to each other, but each is complementary to one of the terminal fragments of a larger oligonucleotide strand that acts as a supramolecular bridge between both particles, due to the high selectivity of the hybridization process. The sizes of the motor and signal particles (around $500 \mathrm{~nm}$ and $10 \mu \mathrm{m}$, resp.) were chosen in such a way that only the second ones will be able to be easily observed by optical microscopy. Thus, if the larger DNA strand (analyte) is not present in the media, the motor particle is not able to capture the signal particle and non detected motion is observable. Employing this system it can be possible to obtain fast information in one single step of the presence of specific oligonucleotide sequences by direct visual inspection of the media using a optical microscopy, which could be a great interest for the development of qualitative analytical devices integrated into lab-on-chip devices for the detection of oligonucleotide sequences characteristic of certain pathogens (Figure 11).

\section{Concluding Remarks}

Ordered mesoporous silica materials have shown an outstanding potential in nanomedicine. Bulk mesoporous silica materials opened the door to drug delivery applications back in 2001, and all the acquired knowledge from that era has been applied to mesoporous silica nanoparticles, which have also been fuelled by the advances in nanotechnology. Mesoporous silica nanoparticles have become excellent nanoplatforms to design smart drug delivery systems for biomedical applications. The development of multifunctional stimuliresponsive drug delivery nanodevices requires the design of nanocarriers addressing biocompatibility and absence of toxicity of all its components, biocompatible trigger stimuli and efficiency to target the suitable cell or tissue, considering the enormous complexity of human body. Significant achievements derived from in vitro experiments have been made during the last decade. In this sense, medicine has benefit from advances in nanotechnology because nanoscale particles have the potential of improving well-established therapeutic approaches. Thanks to the versatility and robustness of silica nanoparticles, multifunctional systems and smart materials have been proposed employing the platform of mesoporous silica nanoparticles. In addition, in vivo findings are encouraging from the perspective of moving the mesoporous silica nanoparticles platforms into clinical trials. Nevertheless, we are just at the beginning of a groundbreaking scientific journey, and much research work remains to be done in this field to allow the transit from bench to bedside.

\section{Acknowledgments}

A special gratitude to my colleagues and friends M. Colilla, M. Manzano, and D Arcos y A Baeza, for their help and sup- 
port in this paper. The author also thanks J. M. Moreno and Pilar Cabañas for his technical help. The author thanks the following for funding this work: the Spanish CICYT through project MAT2008-00736 and the Comunidad Autónoma de Madrid via the S2009MAT-1472 Program Grant. The author would like to express the deepest gratitude to all co-workers and colleagues that have contributed over the years with their effort and thinking to these studies.

\section{References}

[1] T. Yanagisawa, T. Shimizu, K. Kuroda, and C. Kato, "The preparation of alkyltrimethylammonium-kanemite complexes and their conversion to microporous materials," Bulletin of the Chemical Society of Japan, vol. 63, no. 4, pp. 988-992, 1990.

[2] C. T. Kresge, M. E. Leonowicz, W. J. Roth, J. C. Vartuli, and J. S. Beck, "Ordered mesoporous molecular sieves synthesized by a liquid-crystal template mechanism," Nature, vol. 359, no. 6397, pp. 710-712, 1992.

[3] J. S. Beck, J. C. Vartuli, W. J. Roth et al., "A new family of mesoporous molecular sieves prepared with liquid crystal templates," Journal of the American Chemical Society, vol. 114, no. 27, pp. 10834-10843, 1992.

[4] S. Inagaki, S. Guan, T. Ohsuna, and O. Terasaki, "An ordered mesoporous organosilica hybrid material with a crystal-like wall structure," Nature, vol. 416, no. 6878, pp. 304-307, 2002.

[5] Y. Sakamoto, M. Kaneda, O. Terasaki et al., "Direct imaging of the pores and cages of three-dimensional mesoporous materials," Nature, vol. 408, no. 6811, pp. 449-453, 2000.

[6] S. Che, Z. Liu, T. Ohsuna, K. Sakamoto, O. Terasaki, and T. Tatsumi, "Synthesis and characterization of chiral mesoporous silica," Nature, vol. 429, no. 6989, pp. 281-284, 2004.

[7] S. Guan, S. Inagaki, T. Ohsuna, and O. Terasaki, "Cubic hybrid organic-inorganic mesoporous crystal with a decaoctahedral shape," Journal of the American Chemical Society, vol. 122, no. 23, pp. 5660-5661, 2000.

[8] H. X. Deng, S. Grunder, K. E. Cordova et al., "Large-pore apertures in a series of metal-organic frameworks," Science, vol. 336, no. 6084, pp. 1018-1023, 2012.

[9] M. E. Davis, "Ordered porous materials for emerging applications,” Nature, vol. 417, no. 6891, pp. 813-821, 2002.

[10] A. Corma, "From microporous to mesoporous molecular sieve materials and their use in catalysis," Chemical Reviews, vol. 97, no. 6, pp. 2373-2419, 1997.

[11] M. Vallet-Regí, F. Balas, and D. Arcos, "Mesoporous materials for drug delivery," Angewandte Chemie, vol. 46, no. 40, pp. 7548-7558, 2007.

[12] A. Stein, "Advances in microporous and mesoporous solidshighlights of recent progress," Advanced Materials, vol. 15, no. 10 , pp. $763-775,2003$.

[13] M. Manzano and M. Vallet-Regi, "Revisiting bioceramics: bone regenerative and local drug delivery systems," Progress in Solid State Chemistry, vol. 40, no. 3, pp. 17-30, 2012.

[14] S. Inagaki, Y. Fukushima, and K. Kuroda, "Synthesis of highly ordered mesoporous materials from a layered polysilicate," Journal of the Chemical Society, Chemical Communications, no. 8, pp. 680-682, 1993.

[15] D. Zhao, J. Feng, Q. Huo et al., “Triblock copolymer syntheses of mesoporous silica with periodic 50 to 300 angstrom pores," Science, vol. 279, no. 5350, pp. 548-552, 1998.
[16] D. J. De Rosier and A. Klug, "Reconstruction of three dimensional structures from electron micrographs," Nature, vol. 217, no. 5124, pp. 130-134, 1968.

[17] CROWTHER RA, DEROSIER DJ, and KLUG A, "Reconstruction of a three-dimensional structure from projection and its application to electron microscopy," Proceedings of the Royal Society of London, vol. 317, no. 1530, pp. 319-340, 1970.

[18] M. Vallet-Regí, A. Rámila, R. P. Del Real, and J. PérezPariente, "A new property of MCM-41: drug delivery system," Chemistry of Materials, vol. 13, no. 2, pp. 308-311, 2001.

[19] M. Manzano, M. Colilla, and M. Vallet-Reg, "Drug delivery from ordered mesoporous matrices," Expert Opinion on Drug Delivery, vol. 6, no. 12, pp. 1383-1400, 2009.

[20] M. Manzano and M. Vallet-Regí, "New developments in ordered mesoporous materials for drug delivery," Journal of Materials Chemistry, vol. 20, no. 27, pp. 5593-5604, 2010.

[21] F. Balas, M. Manzano, P. Horcajada, and M. Vallet-Regi, "Confinement and controlled release of bisphosphonates on ordered mesoporous silica-based materials," Journal of the American Chemical Society, vol. 128, no. 25, pp. 8116-8117, 2006.

[22] M. Vallet-Regí, "Ordered mesoporous materials in the context of drug delivery systems and bone tissue engineering," Chemistry, vol. 12, no. 23, pp. 5934-5943, 2006.

[23] M. Vallet-Regí, M. Manzano, J. M. González-Calbet, and E. Okunishi, "Evidence of drug confinement into silica mesoporous matrices by STEM spherical aberration corrected microscopy," Chemical Communications, vol. 46, no. 17, pp. 2956-2958, 2010.

[24] F. Hoffmann, M. Cornelius, J. Morell, and M. Fröba, "Silica-based mesoporous organic-inorganic hybrid materials," Angewandte Chemie, vol. 45, no. 20, pp. 3216-3251, 2006.

[25] M. Vallet-Regí, M. Colilla, and B. González, "Medical applications of organic-inorganic hybrid materials within the field of silica-based bioceramics," Chemical Society Reviews, vol. 40, no. 2, pp. 596-607, 2011.

[26] J. C. Doadrio, E. M. B. Sousa, I. Izquierdo-Barba, A. L. Doadrio, J. Perez-Pariente, and M. Vallet-Regí, "Functionalization of mesoporous materials with long alkyl chains as a strategy for controlling drug delivery pattern," Journal of Materials Chemistry, vol. 16, no. 5, pp. 462-466, 2006.

[27] A. Nieto, F. Balas, M. Colilla, M. Manzano, and M. ValletRegí, "Functionalization degree of SBA-15 as key factor to modulate sodium alendronate dosage," Microporous and Mesoporous Materials, vol. 116, no. 1-3, pp. 4-13, 2008.

[28] A. Baeza, E. Guisasola, E. Ruiz-Hernández, and M. ValletRegí, "Magnetically triggered multidrug release by hybrid mesoporous silica nanoparticles," Chemistry of Materials, vol. 24, no. 3, pp. 517-524, 2012.

[29] M. Vallet-Regi and E. Ruiz-Hernandez, "Bioceramics: from bone regeneration to cancer nanomedicine," Advanced Materials, vol. 23, no. 44, pp. 5177-5218, 2011.

[30] M. Vallet-Regí, "Nanostructured mesoporous silica matrices in nanomedicine," Journal of Internal Medicine, vol. 267, no. 1, pp. 22-43, 2010.

[31] D. Arcos, I. Izquierdo-Barba, and M. Vallet-Regí, "Promising trends of bioceramics in the biomaterials field," Journal of Materials Science: Materials in Medicine, vol. 20, no. 2, pp. 447-455, 2009.

[32] D. Arcos and M. Vallet-Regí, "Sol-gel silica-based biomaterials and bone tissue regeneration," Acta Biomaterialia, vol. 6, no. 8, pp. 2874-2888, 2010. 
[33] M. Vallet-Regí, I. Izquierdo-Barba, and M. Colilla, "Structure and functionalization of mesoporous bioceramics for bone tissue regeneration and local drug delivery," Philosophical Transactions of the Royal Society A, vol. 370, pp. 1400-1421, 1963.

[34] F. M. Chen, Y. An, R. Zhang, and M. Zhang, "New insights into and novel applications of release technology for periodontal reconstructive therapies," Journal of Controlled Release, vol. 149, no. 2, pp. 92-110, 2011.

[35] J. L. Vivero-Escoto, I. I. Slowing, V. S. Y. Lin, and B. G. Trewyn, "Mesoporous silica nanoparticles for intracellular controlled drug delivery," Small, vol. 6, no. 18, pp. 19521967, 2010.

[36] Y. Zhao, J. L. Vivero-Escoto, I. I. Slowing, B. G. Trewyn, and V. S. Y. Lin, "Capped mesoporous silica nanoparticles as stimuli-responsive controlled release systems for intracellular drug/gene delivery," Expert Opinion on Drug Delivery, vol. 7, no. 9, pp. 1013-1029, 2010.

[37] J. M. Rosenholm, C. Sahlgren, and M. Lindén, “Towards multifunctional, targeted drug delivery systems using mesoporous silica nanoparticles-opportunities \& challenges," Nanoscale, vol. 2, no. 10, pp. 1870-1883, 2010.

[38] R. Vallet, Mar, H. Ruiz et al., "Design of smart nanomaterials for drug and gene delivery," Journal of Biomaterials and Tissue Engineering, vol. 1, no. 1, pp. 6-29, 2011.

[39] J. Liu, X. Jiang, C. Ashley, and C. J. Brinker, "Electrostatically mediated liposome fusion and lipid exchange with a nanoparticle-supported bilayer for control of surface charge, drug containment, and delivery," Journal of the American Chemical Society, vol. 131, no. 22, pp. 7567-7569, 2009.

[40] C. E. Ashley, E. C. Carnes, G. K. Phillips et al., "The targeted delivery of multicomponent cargos to cancer cells by nanoporous particle-supported lipid bilayers," Nature Materials, vol. 10, no. 5, pp. 389-397, 2011.

[41] M. W. Ambrogio, C. R. Thomas, Y. L. Zhao, J. I. Zink, and J. F. Stoddartt, "Mechanized silica nanoparticles: a new frontier in theranostic nanomedicine," Accounts of Chemical Research, vol. 44, no. 10, pp. 903-913, 2011.

[42] Z. X. Li, J. C. Barnes, A. Bosoy, J. F. Stoddart, and J. I. Zink, "Mesoporous silica nanoparticles in biomedical applications," Chemical Society Reviews, vol. 41, no. 7, pp. 2590-2605, 2012.

[43] J. Khandare, M. Calderon, N. M. Dagia, and R. Haag, "Multifunctional dendritic polymers in nanomedicine: opportunities and challenges," Chemical Society Reviews, vol. 41, no. 7, pp. 2824-2848, 2012.

[44] V. P. Torchilin, "Recent advances with liposomes as pharmaceutical carriers," Nature Reviews Drug Discovery, vol. 4, no. 2, pp. 145-160, 2005.

[45] W. J. M. Mulder, G. J. Strijkers, G. A. F. Van Tilborg, D. P. Cormode, Z. A. Fayad, and K. Nicolay, "Nanoparticulate assemblies of amphiphiles and diagnostically active materials for multimodality imaging," Accounts of Chemical Research, vol. 42, no. 7, pp. 904-914, 2009.

[46] R. Haag and F. Kratz, "Polymer therapeutics: concepts and applications," Angewandte Chemie, vol. 45, no. 8, pp. 1198 $1215,2006$.

[47] K. T. Oh, H. Yin, E. S. Lee, and Y. H. Bae, "Polymeric nanovehicles for anticancer drugs with triggering release mechanisms," Journal of Materials Chemistry, vol. 17, no. 38, pp. 3987-4001, 2007.

[48] Y. Ma, R. J. M. Nolte, and J. J. Cornelissen, "Virus-based nanocarriers for drug delivery," Advanced Drug Delivery Reviews, vol. 64, no. 9, pp. 811-825, 2012.
[49] P. Ghosh, G. Han, M. De, C. K. Kim, and V. M. Rotello, "Gold nanoparticles in delivery applications," Advanced Drug Delivery Reviews, vol. 60, no. 11, pp. 1307-1315, 2008.

[50] A. M. Smith, H. Duan, A. M. Mohs, and S. Nie, "Bioconjugated quantum dots for in vivo molecular and cellular imaging," Advanced Drug Delivery Reviews, vol. 60, no. 11, pp. 1226-1240, 2008.

[51] C. Sun, J. S. H. Lee, and M. Zhang, "Magnetic nanoparticles in MR imaging and drug delivery," Advanced Drug Delivery Reviews, vol. 60, no. 11, pp. 1252-1265, 2008.

[52] E. J. Anglin, L. Cheng, W. R. Freeman, and M. J. Sailor, "Porous silicon in drug delivery devices and materials," Advanced Drug Delivery Reviews, vol. 60, no. 11, pp. 12661277, 2008.

[53] C. Barbé, J. Bartlett, L. Kong et al., "Silica particles: a novel drug-delivery system," Advanced Materials, vol. 16, no. 21, pp. 1959-1966, 2004.

[54] Y. Piao, A. Burns, J. Kim, U. Wiesner, and T. Hyeon, "Designed fabrication of silica-based nanostructured particle systems for nanomedicine applications," Advanced Functional Materials, vol. 18, no. 23, pp. 3745-3758, 2008.

[55] D. Avnir, O. Lev, and J. Livage, "Recent bio-applications of sol-gel materials," Journal of Materials Chemistry, vol. 16, no. 11, pp. 1013-1030, 2006.

[56] W. Tan, K. Wang, X. He et al., "Bionanotechnology based on silica nanoparticles," Medicinal Research Reviews, vol. 24, no. 5, pp. 621-638, 2004.

[57] M. Vallet-Regi and A. Ramila, "New bioactive glass and changes in porosity during the growth of a carbonate hydroxyapatite layer on glass surfaces," Chemistry of Materials, vol. 12, no. 4, pp. 961-965, 2000.

[58] C. E. Ashley, E. C. Carnes, G. K. Phillips et al., "The targeted delivery of multicomponent cargos to cancer cells by nanoporous particle-supported lipid bilayers," Nature Materials, vol. 10, no. 5, pp. 389-397, 2011.

[59] S. H. Wu, Y. Hung, and C. Y. Mou, "Mesoporous silica nanoparticles as nanocarriers," Chemical Communications, vol. 47, no. 36, pp. 9972-9985, 2011.

[60] M. Grün, I. Lauer, and K. K. Unger, "The synthesis of micrometer- and submicrometer-size spheres of ordered mesoporous oxide MCM-41," Advanced Materials, vol. 9, no. 3, pp. 254-257, 1997.

[61] D. Arcos, A. López-Noriega, E. Ruiz-Hernández, O. Terasaki, and M. Vallet-Regí, "Ordered mesoporous microspheres for bone grafting and drug delivery," Chemistry of Materials, vol. 21, no. 6, pp. 1000-1009, 2009.

[62] C. Boissiere, D. Grosso, A. Chaumonnot, L. Nicole, and C. Sanchez, "Aerosol route to functional nanostructured inorganic and hybrid porous materials," Advanced Materials, vol. 23, no. 5, pp. 599-623, 2011.

[63] C. J. Brinker, Y. F. Lu, A. Sellinger, and H. Y. Fan, "Evaporation-induced self-assembly: nanostructures made easy," Advanced Materials, vol. 11, no. 7, p. 579, 1999.

[64] M. Colilla, M. Manzano, I. Izquierdo-Barba, M. Vallet-Reg, C. Boissiére, and C. Sanchez, "Advanced drug delivery vectors with tailored surface properties made of mesoporous binary oxides submicronic spheres," Chemistry of Materials, vol. 22, no. 5, pp. 1821-1830, 2010.

[65] Y. Lu, H. Fan, A. Stump, T. L. Ward, T. Rieker, and C. J. Brinker, "Aerosol-assisted self-assembly of mesostructured spherical nanoparticles," Nature, vol. 398, no. 6724, pp. 223226, 1999.

[66] O. C. Farokhzad and R. Langer, "Impact of nanotechnology on drug delivery," ACS Nano, vol. 3, no. 1, pp. 16-20, 2009. 
[67] Y. S. Lin and C. L. Haynes, "Impacts of mesoporous silica nanoparticle size, pore ordering, and pore integrity on hemolytic activity," Journal of the American Chemical Society, vol. 132, no. 13, pp. 4834-4842, 2010.

[68] W. H. Suh, Y. H. Suh, and G. D. Stucky, "Multifunctional nanosystems at the interface of physical and life sciences," Nano Today, vol. 4, no. 1, pp. 27-36, 2009.

[69] D. Brühwiler, "Postsynthetic functionalization of mesoporous silica," Nanoscale, vol. 2, no. 6, pp. 887-892, 2010.

[70] F. Hoffmann and M. Fröba, "Vitalising porous inorganic silica networks with organic functions - PMOs and related hybrid materials," Chemical Society Reviews, vol. 40, no. 2, pp. 608-620, 2011.

[71] N. Lang and A. Tuel, "A fast and efficient ion-exchange procedure to remove surfactant molecules from MCM-41 materials," Chemistry of Materials, vol. 16, no. 10, pp. 19611966, 2004.

[72] M. C. Burleigh, M. A. Markowitz, M. S. Spector, and B. P. Gaber, "Amine-functionalized periodic mesoporous organosilicas," Chemistry of Materials, vol. 13, no. 12, pp. 4760-4766, 2001.

[73] S. Huh, J. W. Wiench, J. C. Yoo, M. Pruski, and V. S. Y. Lin, "Organic Functionalization and Morphology Control of Mesoporous Silicas via a Co-Condensation Synthesis Method," Chemistry of Materials, vol. 15, no. 22, pp. 42474256, 2003.

[74] M. H. Lim and A. Stein, "Comparative studies of grafting and direct syntheses of inorganic-organic hybrid mesoporous materials," Chemistry of Materials, vol. 11, no. 11, pp. 32853295, 1999.

[75] C. Zapilko, M. Widenmeyer, I. Nagl et al., "Advanced surface functionalization of periodic mesoporous silica: kinetic control by trisilazane reagents," Journal of the American Chemical Society, vol. 128, no. 50, pp. 16266-16276, 2006.

[76] H. Ritter and D. Brühwiler, "Accessibility of amino groups in postsynthetically modified mesoporous silica," Journal of Physical Chemistry C, vol. 113, no. 24, pp. 10667-10674, 2009.

[77] H. P. Lin, L. Y. Yang, C. Y. Mou, S. B. Liu, and H. K. Lee, "A direct surface silyl modification of acid-synthesized mesoporous silica," New Journal of Chemistry, vol. 24, no. 5, pp. 253-255, 2000.

[78] Y. H. Liu, H. P. Lin, and C. Y. Mou, "Direct method for surface silyl functionalization of mesoporous silica," Langmuir, vol. 20, no. 8, pp. 3231-3239, 2004.

[79] E. Ruiz-Hernández, A. López-Noriega, D. Arcos, I. Izquierdo-Barba, O. Terasaki, and M. Vallet-Regí, "Aerosolassisted synthesis of magnetic mesoporous silica spheres for drug targeting," Chemistry of Materials, vol. 19, no. 14, pp. 3455-3463, 2007.

[80] E. Ruiz-Hernández, A. López-Noriega, D. Arcos, and M. Vallet-Regí, "Mesoporous magnetic microspheres for drug targeting," Solid State Sciences, vol. 10, no. 4, pp. 421-426, 2008.

[81] A. P. Alivisatos, "Semiconductor clusters, nanocrystals, and quantum dots," Science, vol. 271, no. 5251, pp. 933-937, 1996.

[82] Y. W. Jun, S. J. Ko, and J. Cheon, "Colloidal Inorganic nanocrystal building blocks," in Nanoparticle Assemblies and Superstructures, N. A. Kotov, Ed., University of Michigan, CRC Press, Ann Arbor, Mich, USA, 2005.
[83] R. F. Ziolo, E. P. Giannelis, B. A. Weinstein et al., "Matrixmediated synthesis of nanocrystalline $\gamma$ - $\mathrm{Fe}_{2} \mathrm{O}_{3}$ : a new optically transparent magnetic material," Science, vol. 257, no. 5067, pp. 219-223, 1992.

[84] D. K. Yi, S. S. Lee, and J. Y. Ying, "Synthesis and applications of magnetic nanocomposite catalysts," Chemistry of Materials, vol. 18, no. 10, pp. 2459-2461, 2006.

[85] Q. A. Pankhurst, J. Connolly, S. K. Jones, and J. Dobson, "Applications of magnetic nanoparticles in biomedicine," Journal of Physics D, vol. 36, no. 13, pp. R167-R181, 2003.

[86] A. Ito, M. Shinkai, H. Honda, and T. Kobayashi, "Medical application of functionalized magnetic nanoparticles," Journal of Bioscience and Bioengineering, vol. 100, no. 1, pp. 1-11, 2005.

[87] A. K. Gupta and M. Gupta, "Synthesis and surface engineering of iron oxide nanoparticles for biomedical applications," Biomaterials, vol. 26, no. 18, pp. 3995-4021, 2005.

[88] C. C. Berry and A. S. G. Curtis, "Functionalisation of magnetic nanoparticles for applications in biomedicine," Journal of Physics D, vol. 36, no. 13, pp. R198-R206, 2003.

[89] L. Neel, "Influence des fluctuations thermiques sur laimantation de grains ferromagnetiques tres fins," Comptes Rendus Hebdomadaires Des Seances De L Academie Des Sciences, vol. 228, no. 8, pp. 664-666, 1949.

[90] S. Mornet, S. Vasseur, F. Grasset, and E. Duguet, "Magnetic nanoparticle design for medical diagnosis and therapy," Journal of Materials Chemistry, vol. 14, no. 14, pp. 2161-2175, 2004.

[91] L. A. Harris, J. D. Goff, A. Y. Carmichael et al., "Magnetite nanoparticle dispersions stabilized with triblock copolymers," Chemistry of Materials, vol. 15, no. 6, pp. 1367-1377, 2003.

[92] T. K. Jain, M. A. Morales, S. K. Sahoo, D. L. Leslie-Pelecky, and V. Labhasetwar, "Iron oxide nanoparticles for sustained delivery of anticancer agents," Molecular Pharmaceutics, vol. 2, no. 3, pp. 194-205, 2005.

[93] A. P. Philipse, M. P. B. Van Bruggen, and C. Pathmamanoharan, "Magnetic silica dispersions: preparation and stability of surface-modified silica particles with a magnetic core," Langmuir, vol. 10, no. 1, pp. 92-99, 1994.

[94] N. Baccile, D. Grosso, and C. Sanchez, "Aerosol generated mesoporous silica particles," Journal of Materials Chemistry, vol. 13, no. 12, pp. 3011-3016, 2003.

[95] B. Muñoz, A. Rámila, J. Pérez-Pariente, I. Díaz, and M. ValletRegí, "MCM-41 organic modification as drug delivery rate regulator," Chemistry of Materials, vol. 15, no. 2, pp. 500-503, 2003.

[96] M. Vallet-Regí, "Revisiting ceramics for medical applications," Dalton Transactions, no. 44, pp. 5211-5220, 2006.

[97] J. Salonen, L. Laitinen, A. M. Kaukonen et al., "Mesoporous silicon microparticles for oral drug delivery: loading and release of five model drugs," Journal of Controlled Release, vol. 108, no. 2-3, pp. 362-374, 2005.

[98] G. V. R. Rao, G. P. Lopez, J. Bravo et al., "Monodisperse mesoporous silica microspheres formed by evaporationinduced self assembly of surfactant templates in aerosols," Advanced Materials, vol. 14, no. 18, p. 1301, 2002.

[99] M. Yoshida and J. Lahann, "Smart nanomaterials," ACS Nano, vol. 2, no. 6, pp. 1101-1107, 2008.

[100] C. C. P. Verstappen, J. J. Heimans, K. Hoekman, and T. J. Postma, "Neurotoxic complications of chemotherapy in patients with cancer: clinical signs and optimal management," Drugs, vol. 63, no. 15, pp. 1549-1563, 2003. 
[101] P. K. Gupta, "Drug targeting in cancer chemotherapy: a clinical perspective," Journal of Pharmaceutical Sciences, vol. 79, no. 11, pp. 949-962, 1990.

[102] M. Ferrari, "Cancer nanotechnology: opportunities and challenges," Nature Reviews Cancer, vol. 5, no. 3, pp. 161-171, 2005.

[103] F. Jotterand, "Nanomedicine: how it could reshape clinical practice," Nanomedicine, vol. 2, no. 4, pp. 401-405, 2007.

[104] S. M. Moghimi, A. C. Hunter, and J. C. Murray, "Nanomedicine: current status and future prospects," FASEB Journal, vol. 19, no. 3, pp. 311-330, 2005.

[105] O. C. Farokhzad and R. Langer, "Nanomedicine: developing smarter therapeutic and diagnostic modalities," Advanced Drug Delivery Reviews, vol. 58, no. 14, pp. 1456-1459, 2006.

[106] A. S. Lübbe, C. Bergemann, J. Brock, and D. G. McClure, "Physiological aspects in magnetic drug-targeting," Journal of Magnetism and Magnetic Materials, vol. 194, no. 1, pp. 149$155,1999$.

[107] B. Hildebrandt, P. Wust, O. Ahlers et al., "The cellular and molecular basis of hyperthermia," Critical Reviews in Oncology/Hematology, vol. 43, no. 1, pp. 33-56, 2002.

[108] J. Overgaard, "Effect of hyperthermia on malignant cells in vivo: a review and a hypothesis," Cancer, vol. 39, no. 6, pp. 2637-2646, 1977.

[109] R. Sharma and C. J. Chen, "Newer nanoparticles in hyperthermia treatment and thermometry," Journal of Nanoparticle Research, vol. 11, no. 3, pp. 671-689, 2009.

[110] A. Jordan, R. Scholz, K. Maier-Hauff et al., "Presentation of a new magnetic field therapy system for the treatment of human solid tumors with magnetic fluid hyperthermia," Journal of Magnetism and Magnetic Materials, vol. 225, no. 1-2, pp. 118-126, 2001.

[111] G. Kong, R. D. Braun, and M. W. Dewhirst, "Hyperthermia enables tumor-specific nanoparticle delivery: effect of particle size," Cancer Research, vol. 60, no. 16, pp. 4440-4445, 2000.

[112] M. Liong, J. Lu, M. Kovochich et al., "Multifunctional inorganic nanoparticles for imaging, targeting, and drug delivery," ACS Nano, vol. 2, no. 5, pp. 889-896, 2008.

[113] R. S. Molday and D. Mackenzie, "Immunospecific ferromagnetic iron-dextran reagents for the labeling and magnetic separation of cells," Journal of Immunological Methods, vol. 52, no. 3, pp. 353-367, 1982.

[114] J. Cheon and J. H. Lee, "Synergistically integrated nanoparticles as multimodal probes for nanobiotechnology," Accounts of Chemical Research, vol. 41, no. 12, pp. 1630-1640, 2008.

[115] B. G. Trewyn, I. I. Slowing, S. Giri, H. T. Chen, and V. S. Y. Lin, "Synthesis and functionalization of a mesoporous silica nanoparticle based on the sol-gel process and applications in controlled release," Accounts of Chemical Research, vol. 40, no. 9, pp. 846-853, 2007.

[116] J. Lu, M. Liong, Z. Li, J. I. Zink, and F. Tamanoi, "Biocompatibility, biodistribution, and drug-delivery efficiency of mesoporous silica nanoparticles for cancer therapy in animals," Small, vol. 6, no. 16, pp. 1794-1805, 2010.

[117] B. G. Trewyn, C. M. Whitman, and V. S. Y. Lin, "Morphological control of room-temperature ionic liquid templated mesoporous silica nanoparticles for controlled release of antibacterial agents," Nano Letters, vol. 4, no. 11, pp. 21392143, 2004.

[118] Y. J. Han, G. D. Stucky, and A. Butler, "Mesoporous silicate sequestration and release of proteins," Journal of the American Chemical Society, vol. 121, no. 42, pp. 9897-9898, 1999.
[119] H. H. P. Yiu, P. A. Wright, and N. P. Botting, "Enzyme immobilisation using siliceous mesoporous molecular sieves," Microporous and Mesoporous Materials, vol. 44-45, pp. 763768, 2001.

[120] S. W. Song, K. Hidajat, and S. Kawi, "Functionalized SBA-15 materials as carriers for controlled drug delivery: influence of surface properties on matrix-drug interactions," Langmuir, vol. 21, no. 21, pp. 9568-9575, 2005.

[121] C. Y. Lai, B. G. Trewyn, D. M. Jeftinija et al., "A mesoporous silica nanosphere-based carrier system with chemically removable CdS nanoparticle caps for stimuli-responsive controlled release of neurotransmitters and drug molecules," Journal of the American Chemical Society, vol. 125, no. 15, pp. 4451-4459, 2003.

[122] D. R. Radu, C. Y. Lai, J. Huang, X. Shu, and V. S. Y. Lin, "Fine-tuning the degree of organic functionalization of mesoporous silica nanosphere materials via an interfacially designed co-condensation method," Chemical Communications, no. 10, pp. 1264-1266, 2005.

[123] T. D. Nguyen, K. C. F. Leung, M. Liong, C. D. Pentecost, J. F. Stoddart, and J. I. Zink, "Construction of a $\mathrm{pH}$-driven supramolecular nanovalve," Organic Letters, vol. 8, no. 15, pp. 3363-3366, 2006.

[124] K. C. F. Leung, T. D. Nguyen, J. F. Stoddart, and J. I. Zink, "Supramolecular nanovalves controlled by proton abstraction and competitive binding," Chemistry of Materials, vol. 18, no. 25, pp. 5919-5928, 2006.

[125] I. I. Slowing, B. G. Trewyn, and V. S. Y. Lin, "Mesoporous silica nanoparticles for intracellular delivery of membraneimpermeable proteins," Journal of the American Chemical Society, vol. 129, no. 28, pp. 8845-8849, 2007.

[126] C. Park, K. Oh, S. C. Lee, and C. Kim, "Controlled release of guest molecules from mesoporous silica particles based on a $\mathrm{pH}$-responsive polypseudorotaxane motif," Angewandte Chemie, vol. 46, no. 9, pp. 1455-1457, 2007.

[127] R. Casasús, E. Climent, M. D. Marcos et al., "Dual aperture control on $\mathrm{pH}$ - and anion-driven supramolecular nanoscopic hybrid gate-like ensembles," Journal of the American Chemical Society, vol. 130, no. 6, pp. 1903-1917, 2008.

[128] S. Angelos, Y. W. Yang, K. Patel, J. F. Stoddart, and J. I. Zink, "pH-responsive supramolecular nanovalves based on cucurbit[6] uril pseudorotaxanes," Angewandte Chemie, vol. 47, no. 12, pp. 2222-2226, 2008.

[129] Q. Fu, G. V. R. Rao, L. K. Ista et al., "Control of molecular transport through stimuli-responsive ordered mesoporous materials," Advanced Materials, vol. 15, no. 15, pp. 12621266, 2003.

[130] R. Hernandez, H. R. Tseng, J. W. Wong, J. F. Stoddart, and J. I. Zink, "An Operational Supramolecular Nanovalve," Journal of the American Chemical Society, vol. 126, no. 11, pp. 33703371, 2004.

[131] T. D. Nguyen, H. R. Tseng, P. C. Celestre et al., "A reversible molecular valve," Proceedings of the National Academy of Sciences of the United States of America, vol. 102, no. 29, pp. 10029-10034, 2005.

[132] T. D. Nguyen, Y. Liu, S. Saha, K. C. F. Leung, J. F. Stoddart, and J. I. Zink, "Design and optimization of molecular nanovalves based on redox-switchable bistable rotaxanes," Journal of the American Chemical Society, vol. 129, no. 3, pp. 626-634, 2007.

[133] K. Patel, S. Angelos, W. R. Dichtel et al., "Enzyme-responsive snap-top covered silica nanocontainers," Journal of the American Chemical Society, vol. 130, no. 8, pp. 2382-2383, 2008. 
[134] N. K. Mal, M. Fujiwara, and Y. Tanaka, "Photocontrolled reversible release of guest molecules from coumarinmodified mesoporous silica," Nature, vol. 421, no. 6921, pp. 350-353, 2003.

[135] S. Angelos, E. Choi, F. Vögtle, L. De Cola, and J. I. Zink, "Photo-driven expulsion of molecules from mesostructured silica nanoparticles," Journal of Physical Chemistry C, vol. 111, no. 18, pp. 6589-6592, 2007.

[136] T. D. Nguyen, K. C. F. Leung, M. Liong, Y. Liu, J. F. Stoddart, and J. I. Zink, "Versatile supramolecular nanovalves reconfigured for light activation," Advanced Functional Materials, vol. 17, no. 13, pp. 2101-2110, 2007.

[137] E. Johansson, E. Choi, S. Angelos, M. Liong, and J. I. Zink, "Light-activated functional mesostructured silica," Journal of Sol-Gel Science and Technology, vol. 46, no. 3, pp. 313-322, 2008.

[138] J. Lu, E. Choi, F. Tamanoi, and J. I. Zink, "Light-activated nanoimpeller-controlled drug release in cancer cells," Small, vol. 4, no. 4, pp. 421-426, 2008.

[139] C. Park, K. Lee, and C. Kim, "Photoresponsive cyclodextrincovered nanocontainers and their sol-gel transition induced by molecular recognition," Angewandte Chemie, vol. 48, no. 7, pp. 1275-1278, 2009.

[140] J. L. Vivero-Escoto, I. I. Slowing, C. W. Wu, and V. S. Y. Lin, "Photoinduced intracellular controlled release drug delivery in human cells by gold-capped mesoporous silica nanosphere," Journal of the American Chemical Society, vol. 131, no. 10, pp. 3462-3463, 2009.

[141] E. Climent, A. Bernardos, R. Martínez-Máñez et al., "Controlled delivery systems using antibody-capped mesoporous nanocontainers," Journal of the American Chemical Society, vol. 131, no. 39, pp. 14075-14080, 2009.

[142] S. Giri, B. G. Trewyn, M. P. Stellmaker, and V. S. Y. Lin, "Stimuli-responsive controlled-release delivery system based on mesoporous silica nanorods capped with magnetic nanoparticles," Angewandte Chemie, vol. 44, no. 32, pp. 50385044, 2005.

[143] E. Ruiz-Hernández, A. Baeza, and M. Vallet-Regí, "Smart drug delivery through DNA/magnetic nanoparticle gates," ACS Nano, vol. 5, no. 2, pp. 1259-1266, 2011.

[144] R. Jin, G. Wu, Z. Li, C. A. Mirkin, and G. C. Schatz, "What controls the melting properties of DNA-linked gold nanoparticle assemblies?" Journal of the American Chemical Society, vol. 125, no. 6, pp. 1643-1654, 2003.

[145] J. Wang and K. M. Manesh, "Motion control at the nanoscale," Small, vol. 6, no. 3, pp. 338-345, 2010.

[146] T. Mirkovic, N. S. Zacharia, G. D. Scholes, and G. A. Ozin, "Fuel for thought: chemically powered nanomotors outswim nature's flagellated bacteria," Acs Nano, vol. 4, no. 4, pp. 1782-1789, 2010.

[147] W. F. Paxton, S. Sundararajan, T. E. Mallouk, and A. Sen, "Chemical locomotion," Angewandte Chemie, vol. 45, no. 33, pp. 5420-5429, 2006.

[148] M. Schliwa and G. Woehlke, "Molecular motors," Nature, vol. 422, no. 6933, pp. 759-765, 2003.

[149] K. J. Böhm, R. Stracke, P. Mühlig, and E. Unger, "Motor protein-driven unidirectional transport of micrometer-sized cargoes across isopolar microtubule arrays," Nanotechnology, vol. 12, no. 3, pp. 238-244, 2001.

[150] R. K. Soong, G. D. Bachand, H. P. Neves, A. G. Olkhovets, H. G. Craighead, and C. D. Montemagno, "Powering an inorganic nanodevice with a biomolecular motor," Science, vol. 290, no. 5496, pp. 1555-1558, 2000.
[151] D. B. Weibel, P. Garstecki, D. Ryan et al., "Microoxen: microorganisms to move microscale loads," Proceedings of the National Academy of Sciences of the United States of America, vol. 102, no. 34, pp. 11963-11967, 2005.

[152] J. R. Howse, R. A. L. Jones, A. J. Ryan, T. Gough, R. Vafabakhsh, and R. Golestanian, "Self-motile colloidal particles: from directed propulsion to random walk," Physical Review Letters, vol. 99, no. 4, Article ID 048102, 2007.

[153] S. J. Ebbens and J. R. Howse, "Direct observation of the direction of motion for spherical catalytic swimmers," Langmuir, vol. 27, no. 20, pp. 12293-12296, 2011.

[154] R. F. Ismagilov, A. Schwartz, N. Bowden, and G. M. Whitesides, "Autonomous movement and self-assembly," Angewandte Chemie, vol. 41, no. 4, p. 652, 2002.

[155] W. F. Paxton, K. C. Kistler, C. C. Olmeda et al., "Catalytic nanomotors: autonomous movement of striped nanorods," Journal of the American Chemical Society, vol. 126, no. 41, pp. 13424-13431, 2004.

[156] S. Fournier-Bidoz, A. C. Arsenault, I. Manners, and G. A. Ozin, "Synthetic self-propelled nanorotors," Chemical Communications, no. 4, pp. 441-443, 2005.

[157] L. Qin, M. J. Banholzer, X. Xu, L. Huang, and C. A. Mirkin, "Rational design and synthesis of catalytically driven nanorotors," Journal of the American Chemical Society, vol. 129, no. 48, pp. 14870-14871, 2007.

[158] R. Laocharoensuk, J. Burdick, and J. Wang, "Carbonnanotube-induced acceleration of catalytic nanomotors," ACS Nano, vol. 2, no. 5, pp. 1069-1075, 2008.

[159] U. K. Demirok, R. Laocharoensuk, K. M. Manesh, and J. Wang, "Ultrafast catalytic alloy nanomotors," Angewandte Chemie, vol. 47, no. 48, pp. 9349-9351, 2008.

[160] P. M. Wheat, N. A. Marine, J. L. Moran, and J. D. Posner, "Rapid fabrication of bimetallic spherical motors," Langmuir, vol. 26, no. 16, pp. 13052-13055, 2010.

[161] J. G. Gibbs and Y. P. Zhao, "Autonomously motile catalytic nanomotors by bubble propulsion," Applied Physics Letters, vol. 94, no. 16, Article ID 163104, 2009.

[162] T. R. Kline, W. F. Paxton, T. E. Mallouk, and A. Sen, "Catalytic nanomotors: remote-controlled autonomous movement of striped metallic nanorods," Angewandte Chemie, vol. 44, no. 5, pp. 744-746, 2005.

[163] J. Burdick, R. Laocharoensuk, P. M. Wheat, J. D. Posner, and J. Wang, "Synthetic nanomotors in microchannel networks: directional microchip motion and controlled manipulation of cargo," Journal of the American Chemical Society, vol. 130, no. 26, pp. 8164-8165, 2008.

[164] A. A. Solovev, Y. Mei, E. B. Ureña, G. Huang, and O. G. Schmidt, "Catalytic microtubular jet engines self-propelled by accumulated gas bubbles," Small, vol. 5, no. 14, pp. 1688$1692,2009$.

[165] K. M. Manesh, M. Cardona, R. Yuan et al., "Templateassisted fabrication of salt-independent catalytic tubular microengines," ACS Nano, vol. 4, no. 4, pp. 1799-1804, 2010.

[166] A. A. Solovev, S. Sanchez, M. Pumera, Y. F. Mei, and O. C. Schmidt, "Magnetic control of tubular catalytic microbots for the transport, assembly, and delivery of micro-objects," Advanced Functional Materials, vol. 20, no. 15, pp. 24302435, 2010.

[167] S. Sanchez, A. A. Solovev, S. M. Harazim, and O. G. Schmidt, "Microbots swimming in the flowing streams of microfluidic channels," Journal of the American Chemical Society, vol. 133, no. 4, pp. 701-703, 2011.

[168] P. Calvo-Marzal, S. Sattayasamitsathit, S. Balasubramanian, J. R. Windmiller, C. Dao, and J. Wang, "Propulsion of nanowire 
diodes," Chemical Communications, vol. 46, no. 10, pp. 1623 1624, 2010.

[169] W. Gao, S. Sattayasamitsathit, K. M. Manesh, D. Weihs, and J. Wang, "Magnetically powered flexible metal nanowire motors," Journal of the American Chemical Society, vol. 132, no. 41, pp. 14403-14405, 2010.

[170] L. Zhang, T. Petit, Y. Lu et al., "Controlled propulsion and cargo transport of rotating nickel nanowires near a patterned solid surface," ACS Nano, vol. 4, no. 10, pp. 6228-6234, 2010.

[171] Y. Wang, R. M. Hernandez, D. J. Bartlett et al., "Bipolar electrochemical mechanism for the propulsion of catalytic nanomotors in hydrogen peroxide solutions," Langmuir, vol. 22, no. 25, pp. 10451-10456, 2006.

[172] S. Sanchez, A. A. Solovev, Y. Mei, and O. G. Schmidt, "Dynamics of biocatalytic microengines mediated by variable friction control," Journal of the American Chemical Society, vol. 132, no. 38, pp. 13144-13145, 2010.

[173] D. Pantarotto, W. R. Browne, and B. L. Feringa, "Autonomous propulsion of carbon nanotubes powered by a multienzyme ensemble," Chemical Communications, no. 13, pp. 15331535, 2008.

[174] N. Mano and A. Heller, "Bioelectrochemical propulsion," Journal of the American Chemical Society, vol. 127, no. 33, pp. 11574-11575, 2005.

[175] D. Kagan, R. Laocharoensuk, M. Zimmerman et al., "Rapid delivery of drug carriers propelled and navigated by catalytic nanoshuttles," Small, vol. 6, no. 23, pp. 2741-2747, 2010.

[176] S. Balasubramanian, D. Kagan, C. M. Jack Hu et al., "Micromachine-enabled capture and isolation of cancer cells in complex media," Angewandte Chemie, vol. 50, no. 18, pp. 4161-4164, 2011.

[177] D. Kagan, S. Campuzano, S. Balasubramanian, F. Kuralay, G. U. Flechsig, and J. Wang, "Functionalized micromachines for selective and rapid isolation of nucleic acid targets from complex samples," Nano Letters, vol. 11, no. 5, pp. 2083-2087, 2011.

[178] D. Kagan, P. Calvo-Marzal, S. Balasubramanian et al., "Chemical sensing based on catalytic nanomotors: motionbased detection of trace silver," Journal of the American Chemical Society, vol. 131, no. 34, pp. 12082-12083, 2009.

[179] J. Wu, S. Balasubramanian, D. Kagan, K. M. Manesh, S. Campuzano, and J. Wang, "Motion-based DNA detection using catalytic nanomotors," Nature Communications, vol. 1, no. 4 , article $36,2010$.

[180] J. Simmchen, A. Baeza, D. Ruiz, M. J. Esplandiu, and M. VALLET REGÍ, "Asymmetric hybrid silica nanomotors for capture and cargo transpotrt: towards a novel motion-based DNA," Small, vol. 8, no. 13, pp. 2053-2059, 2012. 

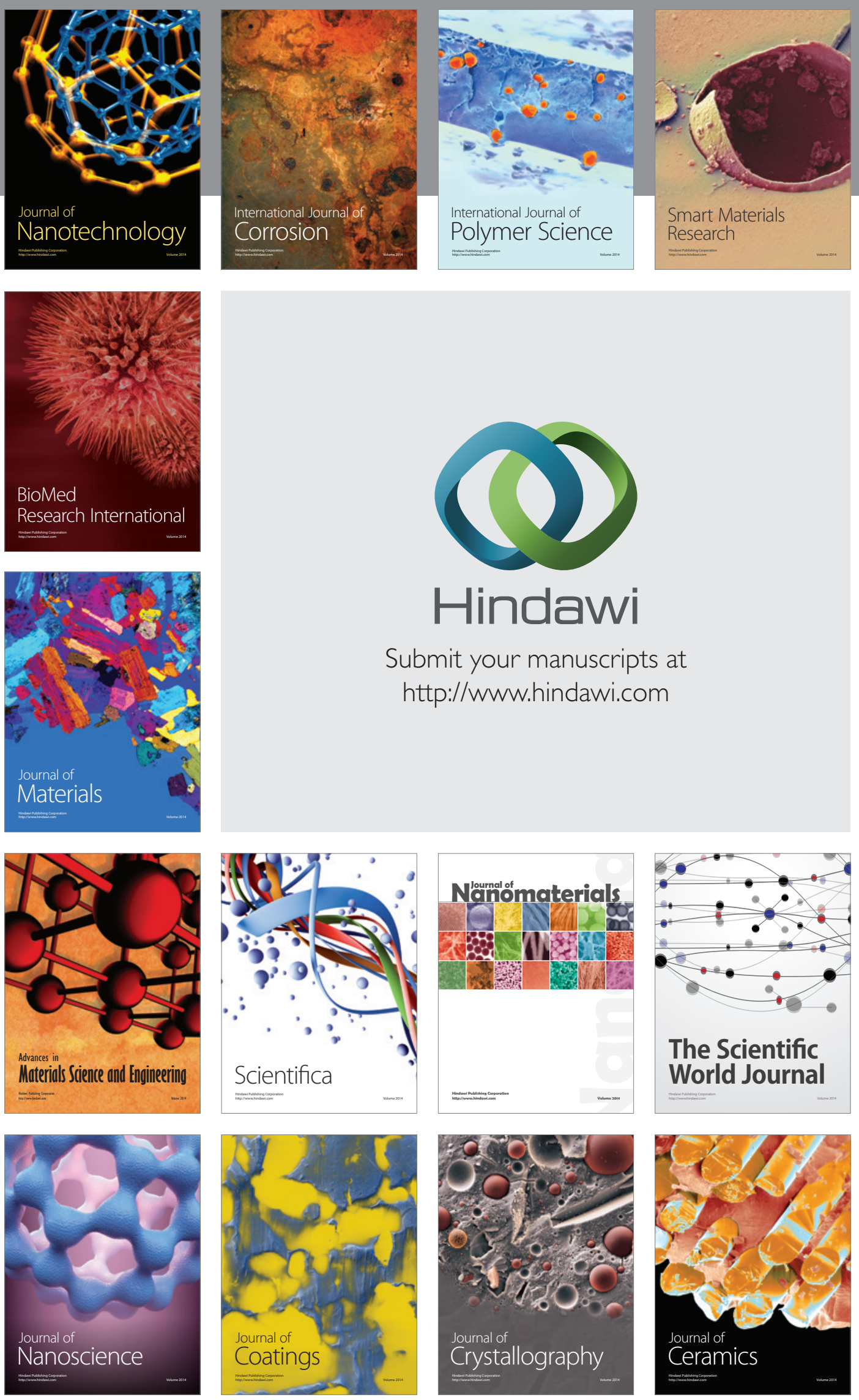

The Scientific World Journal

Submit your manuscripts at

http://www.hindawi.com

\section{World Journal}

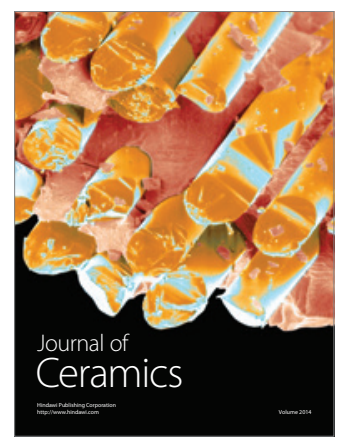

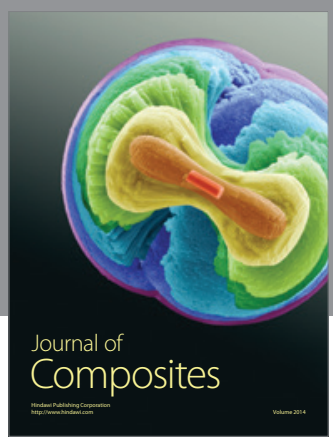
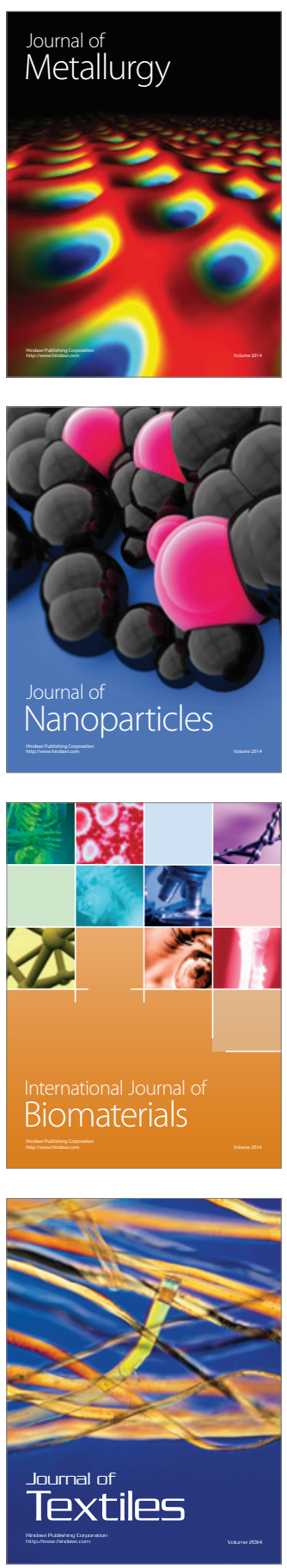